TRANSACTIONS OF THE

AMERICAN MATHEMATICAL SOCIETY

Volume 352, Number 8, Pages 3651-3685

S 0002-9947(00)02112-7

Article electronically published on April 17, 2000

\title{
MULTISCALE DECOMPOSITIONS ON BOUNDED DOMAINS
}

\author{
A. COHEN, W. DAHMEN, AND R. DEVORE
}

\begin{abstract}
A construction of multiscale decompositions relative to domains $\Omega \subset \mathbb{R}^{d}$ is given. Multiscale spaces are constructed on $\Omega$ which retain the important features of univariate multiresolution analysis including local polynomial reproduction and locally supported, stable bases.
\end{abstract}

\section{INTRODUCTION}

Multiscale methods have become an important and powerful tool in several areas of mathematical analysis and applications. Since the introduction of wavelet bases, the interest in these methods has grown in a large scientific community.

Recall that wavelet bases are usually constructed with the aid of multiresolution analysis. In the univariate case, multiresolution for $L_{2}(\mathbb{R})$ is given by an ascending sequence

$$
\cdots \subset V_{-1} \subset V_{0} \subset V_{1} \subset V_{2} \subset \cdots L_{2}(\mathbb{R})
$$

of closed subspaces $V_{j}$ of $L_{2}(\mathbb{R})$ that satisfy

$$
\bigcap_{j \in \mathbb{Z}} V_{j}=\{0\}, \quad \overline{\bigcup_{j \in \mathbb{Z}} V_{j}}=L_{2}(\mathbb{R}),
$$

where the closure is taken with respect to the $L_{2}(\mathbb{R})$-norm. Moreover, each $V_{j}$ has the form $V_{j}=\overline{\operatorname{span}\left\{\varphi_{j, k}: k \in \mathbb{Z}\right\}}$ where $\varphi_{j, k}:=2^{j / 2} \varphi\left(2^{j} \cdot-k\right)$ with $\varphi$ a fixed function from $L_{2}(\mathbb{R})$.

The function $\varphi$ is assumed to satisfy a refinement (or scaling) equation

$$
\varphi(x)=\sqrt{2} \sum_{k \in \mathbb{Z}} h_{k} \varphi(2 x-k)
$$

which, in particular, guarantees the nestedness of the spaces $V_{j}$. The generator $\varphi$ can also be chosen to satisfy additional properties which are important in applications. For example, $\varphi$ will have compact support if the scaling coefficients $h_{k}$ appearing in (1.1) are finite in number. Also, Daubechies [Dau] has constructed generators $\varphi$ of compact support whose integer shifts $\varphi(\cdot-k), k \in \mathbb{Z}$, are orthonormal.

In this paper, we shall enlarge our attention to the case of biorthogonal wavelets as described in Cohen, Daubechies, and Feauveau [CDF]. In this case, the refinable

Received by the editors December 28, 1995 and, in revised form, February 26, 1997.

2000 Mathematics Subject Classification. Primary 41A63, 42C15.

This research was supported by ONR Contract N0014-91-J1343 and a NATO travel grant.

(C)2000 American Mathematical Society 
function $\varphi$ has a dual function $\tilde{\varphi}$, i.e.,

$$
\int_{\mathbb{R}} \varphi(x) \tilde{\varphi}(x-k) d x=\delta_{0, k}, \quad k \in \mathbb{Z},
$$

which is also refinable. Examples of such dual pairs of refinable functions can be found in $\overline{\mathrm{CDF}}$.

Multiresolution analysis is used to construct a wavelet function $\psi$ that encodes the details between any two successive levels of resolution in the sense that $\left\{\psi_{j, k}: k \in \mathbb{Z}\right\}$ spans a complementing space $W_{j}$ of $V_{j}$ in $V_{j+1}$. The first construction of wavelets yielded functions $\psi$ (called orthonormal wavelets) whose shifts are orthogonal. In this case, the family of functions $\left\{\psi_{j, k}\right\}_{j, k \in \mathbb{Z}}$ is an orthonormal basis for $L_{2}(\mathbb{R})$.

In our setting of biorthogonal wavelets, multiresolution gives a pair of functions $\psi$ and $\tilde{\psi}$ which are in duality

$$
\int_{\mathbb{R}} \psi(x) \tilde{\psi}(x-k)=\delta_{0, k} .
$$

Then, $\left\{\psi_{j, k}\right\}_{j, k \in \mathbb{Z}}$ is a Riesz basis for $L_{2}(\mathbb{R})$ and $\left\{\tilde{\psi}_{j, k}\right\}_{j, k \in \mathbb{Z}}$ is its dual basis. This means that each function $f \in L_{2}(\mathbb{R})$ has a unique wavelet decomposition

$$
f=\sum_{j, k \in \mathbb{Z}} c_{j, k}(f) \psi_{j, k}, \quad c_{j, k}(f)=\int_{\mathbb{R}} f(x) \overline{\tilde{\psi}_{j, k}(x)} d x
$$

with convergence in the sense of $L_{2}(\mathbb{R})$, and the following stability estimates hold

$$
c_{1} \sum\left|c_{j, k}(f)\right|^{2} \leq\|f\|_{L_{2}(\mathbb{R})}^{2} \leq c_{2} \sum\left|c_{j, k}(f)\right|^{2}
$$

with $c_{1}$ and $c_{2}$ positive constants.

There are several important features that make wavelets particularly attractive in both theory and practice. We mention some of these.

- The wavelet decomposition is local in both time and frequency. In particular, if $\varphi$ and $\psi$ are compactly supported, the decomposition (1.2) at a point $x$ only involves those functions whose support contains $x$.

- The wavelet decomposition of a sampled function can be efficiently computed by the Fast Wavelet Transform when the generator $\varphi$ and the wavelet $\psi$ have compact support.

- Various functions spaces can be characterized in terms of the wavelet coefficients. For example, the Besov spaces $B_{q}^{s}\left(L_{p}(\mathbb{R})\right)$ (including the generalized Lipschitz classes $B_{\infty}^{s}\left(L_{\infty}(\mathbb{R})\right)$ and the Sobolev classes $\left.B_{2}^{s}\left(L_{2}(\mathbb{R})\right)\right)$ are characterized by weighted sequence norms of the coefficients $c_{j, k}(f)$ in (1.2). This characterization holds for some range of the smoothness parameter $s \in \mathbb{R}$ depending on the regularity of $\varphi$ and the largest $N$ for which all polynomials of degree $N$ are contained in $V_{0}$.

Multiresolution analysis and its advantages extend rather easily to the functions defined on $\mathbb{R}^{d}$, by means of tensor products preserving all the above mentioned important features. However, in many applications, multiscale decompositions of functions are required relative to some bounded domain $\Omega \subset \mathbb{R}^{d}$. This need arises, for instance, when partitioning images as well as when employing multiscale techniques for the solution of operator equations, or when characterizing Besov spaces on bounded domains. It is clear that simply restricting scaling functions and wavelets defined on all of $\mathbb{R}^{d}$ to $\Omega$ would in general destroy orthogonality and 
stability. Moreover, straightforward reorthonormalization might be unstable and destroy locality. The main purpose of the present paper is to develop a multiresolution analysis for domains $\Omega$ which preserves the important properties mentioned above.

In order to motivate the multivariate constructions given later in this paper, it is useful to recall the techniques that have been proposed to handle multiresolution in the univariate case (see e.g. [CDV, $\mathrm{CQ}$ ). The approach in $\mathrm{CQ}$ makes use of $B$ spline techniques and therefore seems to be confined to piecewise polynomial scaling functions. Moreover, it doesn't seem to permit an extension to the multivariate case except for rectangular domains whose boundaries coincide with grid lines.

The approach in $[\mathrm{CDV}]$ is similar to $[\mathrm{CQ}$ in that one tries to preserve as many features of multiresolution on $\mathbb{R}$ as possible. A ladder of spaces

$$
V_{1}([0,1]) \subset V_{1}([0,1]) \subset \cdots \subset L_{2}([0,1])
$$

is constructed for $[0,1]$ which on a large subset of $[0,1]$ agrees with a multiresolution analysis for $\mathbb{R}$ generated by some scaling function $\varphi$ as in (1.1). Assuming that $\varphi$ has compact support, each $V_{j}([0,1])$ (for $j$ sufficiently large) is spanned by the functions

$$
\varphi\left(2^{j} \cdot-k\right), \quad 2^{-j} k \in \Omega_{0}^{j}
$$

for some set $\Omega_{0}^{j} \subset 2^{-j} \mathbb{Z}$ such that $2^{-j} k \in \Omega_{0}^{j}$ implies $\operatorname{supp}\left(\varphi\left(2^{j} \cdot-k\right)\right) \subset[0,1]$, and by certain modified basis functions $\varphi_{j, n}^{0}, \varphi_{j, n}^{1}, n=0, \ldots, N$, supported near the end points of the interval. Here, $N$ is again the degree of polynomials that should be contained in $V_{j}([0,1])$. Thus, $V_{j}([0,1])$ is the restriction to $[0,1]$ of a certain subspace of the space $V_{j}(\mathbb{R})$ spanned by the $\varphi\left(2^{j} \cdot-k\right), k \in \mathbb{Z}$.

The basic idea from [CDV] for constructing the boundary functions $\varphi_{j, n}^{i}, i=0,1$, $n=0, \ldots, N$, can be described as follows. Suppose that $\varphi$ has compact support. Then there is an integer $L$ such that

$$
\operatorname{supp}(\varphi) \subseteq[-L, L]
$$

Our assumption on the refinability of $\varphi$ imply that $\hat{\varphi}(0) \neq 0$. Suppose further that $\varphi$ satisfies Strang-Fix conditions of order $N$, i.e., the Fourier transform $\hat{\varphi}$ of $\varphi$ satisfies

$$
\hat{\varphi}^{(n)}=0, \quad k \in \mathbb{Z} \backslash\{0\}, \quad n=0, \ldots, N .
$$

It is well-known (and firstly proved in [Sch]) that (1.5) implies the existence of polynomials $p_{n}$ such that, if we set $p_{n, k}=p_{n}(k)$, we have

$$
x^{n}=\sum_{k \in \mathbb{Z}} p_{n, k} \varphi(n-k), \quad n=0, \ldots, N .
$$

It follows that the monomial $x^{n}$ is locally in $V_{0}$ and the coefficients $p_{n, k}$ are therefore given by

$$
p_{n, k}=\int_{\mathbb{R}} x^{n} \overline{\tilde{\varphi}(x-k)} d x
$$


Then, we can define for $n=0, \ldots, N, p_{n, k}^{j}=2^{-j n} p_{n, k}$, and for all $x \in[0,1]$,

$$
\begin{gathered}
\varphi_{j, n}^{0}(x)=x^{n}-\sum_{k=L}^{+\infty} p_{n, k}^{j} \varphi\left(2^{j} x-k\right)=2^{-j / 2} \sum_{k=-L+1}^{L-1} p_{n, k}^{j} \varphi_{j, k}, \\
\varphi_{j, n}^{1}(x)=x^{n}-\sum_{k=-\infty}^{2^{j}-L} p_{n, k}^{j} \varphi\left(2^{j} x-k\right)=2^{-j / 2} \sum_{k=2^{j}-L+1}^{2^{j}+L-1} p_{n, k}^{j} \varphi_{j, k} .
\end{gathered}
$$

We generate the space $V_{j}([0,1])$ by the interior functions $\varphi_{j, k}, k=L, \ldots, 2^{j}-L$, and the boundary functions $\varphi_{j, k}^{i}, i=0,1, k=0, \ldots, N$.

The following facts are then easily verified.

(i) $\operatorname{diam}\left(\operatorname{supp}\left(\varphi_{j, n}^{i}\right)\right) \sim \operatorname{diam}\left(\operatorname{supp}\left(\varphi_{j, k}\right)\right) \sim 2^{-j}$.

(ii) $\Pi_{N}([0,1]) \subseteq V_{j}([0,1])$ for $j \in \mathbb{N}$, where $\Pi_{N}([0,1])$ denotes the space of all polynomials on $[0,1]$ of degree at most $N$.

(iii) $V_{j}([0,1]) \subset V_{j+1}([0,1])$ for $j \in \mathbb{N}$.

Here, the support of these functions is taken on $[0,1]$. Property (iii) is a consequence of (1.7) and the fact that the interior functions $\varphi_{j, k}, i=L, \ldots, 2^{j}-L$, are refinable by (1.1).

The purpose of the present paper is to develop a similar strategy for constructing multiresolution spaces $V_{j}(\Omega)$ relative to a given bounded domain $\Omega \subset \mathbb{R}^{d}$. We shall also study the corresponding multiscale decompositions of various function spaces defined on $\Omega$. Our goal is to not have our construction restricted to rectangular domains (or unions of these) but to apply to general domains $\Omega$ whose boundary $\partial \Omega$ has sufficient regularity.

It is easy to see that the ideas described above for the univariate case will not carry over to the multivariate case in a straightforward manner. For example, suppose that $\phi \in L_{2}\left(\mathbb{R}^{d}\right)$ is a refinable function of compact support and that the multivariate polynomials $x^{\beta}$ admit an expansion

$$
x^{\beta}=\sum_{k \in \mathbb{Z}^{d}} p_{\beta, k}^{j} \phi\left(2^{j} x-k\right) .
$$

In analogy to the univariate case, we may define a space $V_{j}(\Omega)$ as the span of functions $\phi\left(2^{j} \cdot-k\right)$ whose support is strictly contained in $\Omega$, together with the functions

$$
x^{\beta}-\sum_{k \in \Omega_{j}} p_{\beta, k}^{j} \phi\left(2^{j} x-k\right)
$$

where $\Omega_{j}$ consists of such $k \in \mathbb{Z}^{d}$ such that $\operatorname{supp}\left(\phi\left(2^{-j} \cdot-k\right)\right) \subset \Omega$. However, these latter functions will be localized only in directions pointing into the domain but not in directions tangential to the boundary. To obtain a ladder of spaces $V_{j}(\Omega)$, $j=0,1, \ldots$, whose spanning functions are completely localized and to retain the other desired properties of multiresolution will take much more sophisticated considerations. The main difficulty to be overcome is how to construct suitable linear combinations of the $\phi\left(2^{j} \cdot-k\right)$ near the boundary so that the following properties hold:

(I) The functions spanning $V_{j}(\Omega)$ all have compact support and their diameters are of the order $2^{-j}$

(II) The functions spanning $V_{j}(\Omega)$ are refinable, i.e., they can be expressed in terms of the functions spanning $V_{j+1}(\Omega)$; 
(III) The resulting linear span $V_{j}(\Omega)$ contains all the polynomials up to a certain degree.

In $₫ 2$ we formulate conditions on a domain $\Omega$ which allow the construction of a ladder of spaces $V_{j}(\Omega), j \geq 0$, whose spanning functions satisfy properties (I)-(III). Our conditions are stated in terms of the existence of partitions of the lattice points $2^{-j} \mathbb{Z}^{d}$ relative to $\Omega$. In 93 , we show how to construct spaces $V_{j}(\Omega)$, $j=0,1, \ldots$, satisfying (I)-(III) given that the conditions of $\$ 2$ are valid for $\Omega$. \$4 is concerned with corresponding multiscale decompositions of function spaces. Specifically we establish frame bounds, provide characterizations of Besov spaces on $\Omega$, and establish some of the elements of Littlewood-Paley theory for our multiscale decompositions.

For many specific domains it is relatively easy to verify that they satisfy the conditions of $\$ 2$ and therefore permit multiresolution and all the ensuant properties of $\S 93$, 4. It is also possible, but substantially more difficult, to show that general classes of domains satisfy the conditions of $\$ 2$ In $\$ 5$ we verify this for domains $\Omega \subset \mathbb{R}^{2}$ whose boundary has certain piecewise Lipschitz smoothness.

One of the major interest of our approach is to provide simple algorithms for multiscale decomposition adapted to bounded domains. We have chosen to describe these algorithms and concrete examples in a separate work. The main goal of the present paper is to provide the theoretical setting of the underlying multiresolution analysis and study its properties with respect to function spaces.

\section{A GENERAL FORMAT FOR THE CONSTRUCTION OF BOUNDARY FiLETS}

We give in this section, sufficient conditions on a domain $\Omega$ and a multivariate scaling function $\phi$ in order that a multiresolution on $\Omega$ can be constructed. The conditions we impose on $\Omega$ are in the form of the existence of partitions of certain sets $\Omega_{j} \subset 2^{-j} \mathbb{Z}^{d}, j \geq 0$, associated to $\Omega$ and $\phi$. In $\$ 5$ we show (in the case of two space dimensions) how to verify these assumptions for a general class of domains.

Multiresolution is well understood when the underlying domain is $\mathbb{R}^{d}$. Since this is also our starting point for the construction of multiresolution on domains $\Omega$, we shall briefly review this construction. For more details the reader can consult BDR, Dau2, JM, Me.

Let $\phi \in L^{2}\left(\mathbb{R}^{d}\right)$ be a function which satisfies the refinement equation

$$
\phi(x)=2^{d / 2} \sum_{k \in \mathbb{Z}^{d}} a_{k} \phi(2 x-k) .
$$

In the following we will have to make use of several coordinate properties of $\phi$. Therefore we shall restrict ourselves to the case that $\phi$ is the tensor product of a univariate scaling function $\varphi$ :

$$
\phi\left(x_{1}, \ldots, x_{d}\right):=\varphi\left(x_{1}\right) \cdots \varphi\left(x_{d}\right) .
$$

Concerning the univariate scaling function $\varphi$, we shall assume that $\varphi$ satisfies the following properties:

(P1) $\operatorname{supp}(\varphi) \subset\left[N_{1}, N_{2}\right]$ for some $N_{1}, N_{2} \in \mathbb{Z}$ and $\varphi$ is not identically zero on $[0,1]$. (P2) $\varphi$ satisfies (11.1) with $h_{k}=0$ if $k<N_{1}$ or $k>N_{2}$ and $h_{N_{1}} h_{N_{2}} \neq 0$.

(P3) There exists a compactly supported function $\tilde{\varphi} \in L^{2}(\mathbb{R})$ which is also refinable

$$
\tilde{\varphi}(x)=\sqrt{2} \sum_{k \in \mathbb{Z}} \tilde{h}(k) \tilde{\varphi}(2 x-k),
$$


and satisfies the biorthogonality relation

$$
\int_{\mathbb{R}} \varphi(x) \tilde{\varphi}(x-k) d x=\delta_{k, 0}, \quad k \in \mathbb{Z} .
$$

(P4) $\varphi$ satisfies the Strang-Fix conditions (1.5) of order $N$. This means that each univariate polynomial $P$ of degree at most $N$ can be represented as a linear combination of the shifts $\varphi(\cdot-k), k \in \mathbb{Z}$. More precisely, when $\varphi$ is normalized by $\int_{\mathbb{R}} \varphi(x) d x=1$, one has (cf. [CDM] that for every polynomial $P$ in $\Pi_{n}$, $n \leq N$,

$$
P(x)-\sum_{k \in \mathbb{Z}} P(k) \varphi(x-k) \in \Pi_{n-1} .
$$

The assumptions (P1-4) are the usual starting point for the construction of univariate biorthogonal wavelets [CDF]. In particular, under these assumptions there exist biorthogonal wavelets

$$
\psi(x)=\sum_{n \in \mathbb{Z}}(-1)^{n} \tilde{h}_{1-n} \varphi(2 x-n), \quad \tilde{\psi}(x)=\sum_{n \in \mathbb{Z}}(-1)^{n} h_{1-n} \tilde{\varphi}(2 x-n),
$$

which satisfy

$$
\int_{\mathbb{R}} \varphi(x) \tilde{\psi}(x-k) d x=\int_{\mathbb{R}} \tilde{\varphi}(x) \psi(x-k) d x=0, \quad k \in \mathbb{Z},
$$

and

$$
\int_{\mathbb{R}} \psi(x) \tilde{\psi}(x-k) d x=\delta_{0, k}, \quad k \in \mathbb{Z} .
$$

Since these functions all have compact support, there is an integer $L$ such that

$$
\operatorname{supp}(\varphi), \operatorname{supp}(\tilde{\varphi}), \operatorname{supp}(\psi), \operatorname{supp}(\tilde{\psi}) \subseteq[-L, L] .
$$

Remark 2.1. If $\varphi, \tilde{\varphi}$ are refinable dual generators (as described in (P3)) with compact support, then their masks have finite support so that the conditions on the coefficients $h_{k}$ in (P2) are automatically satisfied. Indeed, from (2.3), it follows that only finitely many of the coefficients $h_{k}$ are different from zero. The assertion then follows by comparing supports on both sides of (1.1).

Remark 2.2. It is easy to see that (2.3) implies stability of the shifts $\varphi(\cdot-k), k \in \mathbb{Z}$ (and likewise of the $\tilde{\varphi}(\cdot-k)$ ), by which we mean that there exist positive constants $c_{1}, c_{2}$ such that

$$
c_{1}\left(\sum_{k \in \mathbb{Z}}\left|\lambda_{k}\right|^{2}\right)^{1 / 2} \leq\left\|\sum_{k \in \mathbb{Z}} \lambda_{k} \varphi_{j, k}\right\|_{L^{2}(\mathbb{R})} \leq c_{2}\left(\sum_{k \in \mathbb{Z}}\left|\lambda_{k}\right|^{2}\right)^{1 / 2},
$$

holds for all sequences $\left\{\lambda_{k}\right\}_{k \in \mathbb{Z}}$ in $l^{2}(\mathbb{Z})$. Note that these constants are independent of the scale parameter $j$.

The tensor product $\phi$ of (2.2) satisfies the two-scale relation (2.1), with coefficients $a_{k}=h_{k_{1}} \cdots h_{k_{d}}, k=\left(k_{1}, \ldots, k_{d}\right) \in \mathbb{Z}^{d}$. Likewise, for $\tilde{\phi}(x):=\tilde{\varphi}\left(x_{1}\right) \cdots \tilde{\varphi}\left(x_{d}\right)$, the (multi-) integer shifts $\phi(\cdot-k), k \in \mathbb{Z}^{d}$, are biorthogonal to $\phi$ in the sense of (2.3). Consequently, the shifts $\phi(\cdot-k), \tilde{\phi}(\cdot-k), k \in \mathbb{Z}^{d}$, are again stable with respect to the obvious analogous notion of stability on $\mathbb{Z}^{d}$.

We shall use the standard multi-index notation $x^{\alpha}:=x_{1}^{\alpha_{1}} \cdots x_{d}^{\alpha_{d}}$ and the notation $\alpha \leq N$ to mean $\alpha_{i} \leq N, i=1, \ldots, d$. We denote by $\Pi_{N}$ the space of all 
polynomials $P(x)=\sum_{0 \leq \alpha \leq N} c_{\alpha} x^{\alpha}$ of coordinate degree $N$. It follows from (P4), that for each $P \in \Pi_{N}$, there exists a unique polynomial $Q$ of lower total degree such that

$$
P(x)=\sum_{k \in \mathbb{Z}^{d}} P(k) \phi(x-k)+Q(x) .
$$

The spaces

$$
V_{j}=\overline{\operatorname{span}\left\{\phi\left(2^{j} \cdot-k\right): k \in \mathbb{Z}^{d}\right\}}
$$

form a multiresolution analysis for $L_{2}\left(\mathbb{R}^{d}\right)$ and contain $\Pi_{N}$ with $N$ as in (P4).

Given a domain $\Omega \subset \mathbb{R}^{d}$, we would like to construct suitable subspaces of $V_{j}$ whose restrictions $V_{j}(\Omega)$ to $\Omega$ form a multiresolution of $L^{2}(\Omega)$ :

$$
V_{0}(\Omega) \subset V_{1}(\Omega) \subset \cdots \subset L_{2}(\Omega), \quad \overline{\bigcup_{j=0}^{\infty} V_{j}(\Omega)}=L_{2}(\Omega),
$$

and contain the space $\Pi_{N}(\Omega)$ of all polynomials of coordinate degree $N$ on $\Omega$.

In order to motivate the construction of the spaces $V_{j}(\Omega)$ that follows, it will be useful to consider the following simple but instructive example with $\Omega=[0,1] \times$ $[0,1] \subset \mathbb{R}^{2}$. In this case, the multiresolution spaces can be simply constructed as tensor products

$$
V_{j}(\Omega)=V_{j}([0,1]) \otimes V_{j}([0,1]),
$$

where the $V_{j}([0,1])$ are the spaces for $[0,1]$ as described in the introduction. The generators of $V_{j}(\Omega)$ are of the following three types:

a) The tensor product of two interior scaling functions, i.e., functions whose supports are fully contained in $[0,1]$, for instance,

$$
2^{j} \varphi\left(2^{j} x-k\right) \varphi\left(2^{j} y-m\right), \quad L \leq k, m \leq 2^{j}-L .
$$

b) The tensor product of an interior scaling function and a boundary scaling function, such as

$$
\varphi_{j, n}^{0}(x) \varphi_{j, k}(y)=2^{j} \sum_{m=-L+1}^{L-1} P_{n}(m) \varphi\left(2^{j} x-m\right) \varphi\left(2^{j} y-k\right),
$$

for $L \leq k \leq 2^{j}-L, 0 \leq n \leq N$.

c) The tensor product of two boundary scaling functions, e.g., for $0 \leq n_{1}, n_{2}$ $\leq N$,

$$
\varphi_{j, n_{1}}^{0}(x) \varphi_{j, n_{2}}^{0}(y)=2^{j} \sum_{-L+1 \leq k, m \leq L} P_{n_{1}}(k) P_{n_{2}}(m) \varphi\left(2^{j} x-k\right) \varphi\left(2^{j} y-m\right) .
$$

Note that these three types of functions can be distinguished by the dimension of the sets of lattice points involved in their defining linear combinations. In a) no linear combination is taken. Each basis function is associated with a single lattice point, a zero-dimensional set. In b) only linear combinations with respect to finitely many lattice points in one coordinate direction are involved which corresponds to a onedimensional set of lattice points. The last group c) corresponds to two-dimensional sets of lattice points.

This is illustrated in Figure 1 where functions of type a) are represented by single lattice points, those of type b) by horizontal or vertical "towers" of lattice points, and those of type c) by rectangular arrays of lattice points in the "corners". Note 


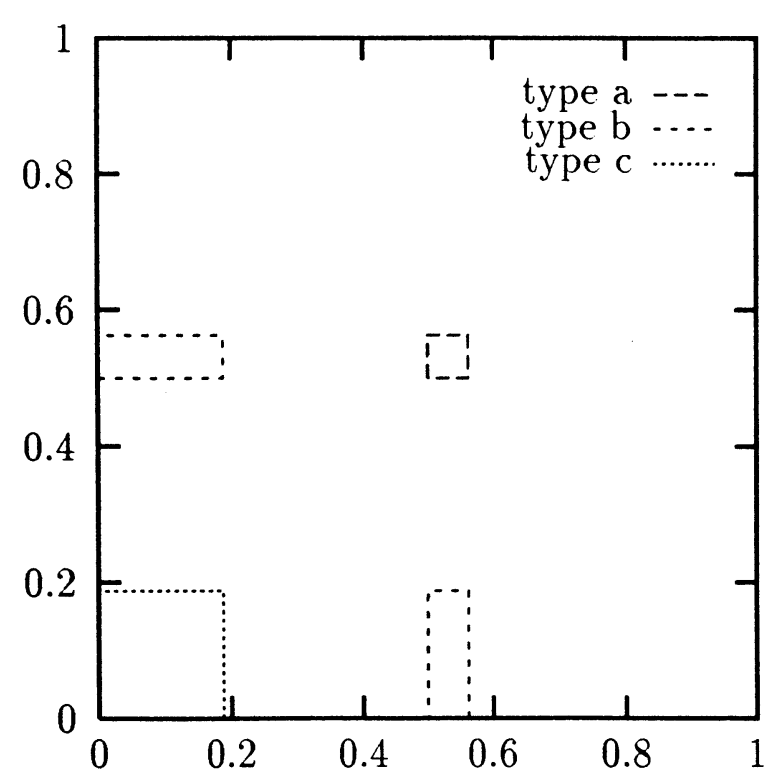

FIGURE 1. Supports of scaling functions of different types

also that further distinctions can be made in terms of orientation. For instance, vertical towers can be oriented upward or downward.

Our strategy for constructing $V_{j}(\Omega)$ for more general domains $\Omega$ will be similar to the case $[0,1] \times[0,1]$ just discussed. The space $V_{j}(\Omega)$ will be spanned by a collection of basis functions. Each basis function is associated to a set, which we call a cell, of lattice points from $2^{-j} \mathbb{Z}^{d}$. A cell will have a spatial dimension which will be given by a set $I \subset\{1, \ldots, d\}$ and a direction which will be given by a vector $\left(\sigma_{i}\right)_{i \in I} \subset\{-1,1\}^{|I|}$. Each dyadic level $j$ will have a collection of cells $\mathcal{C}_{j}$ and a set of basis functions for $V_{j}(\Omega)$. The existence of a multiresolution $V_{j}(\Omega)$ for $\Omega$ with the properties we desire will rest upon properties of the collections $\mathcal{C}_{j}$. In what remains of this section, we shall formulate sufficient conditions on the collections $\mathcal{C}_{j}$ to guarantee the existence of a multiresolution for $\Omega$ with the desired properties. Later (in 95 ), we shall give classes of domains in $\mathbb{R}^{2}$ which satisfy these conditions.

Let $\Omega \subset \mathbb{R}^{d}$ be a bounded open domain which will be fixed in this and the next section. For each $j=0,1, \ldots$, we let

$$
\Omega_{j}:=\left\{2^{-j} k: k \in \mathbb{Z}^{d}, \Omega \cap 2^{-j}\left(k+[-L, L]^{d}\right) \neq \varnothing\right\}
$$

denote the set of lattice points for which the support of $\phi\left(2^{j} \cdot-k\right)$ may intersect $\Omega$. Furthermore, for each $j=0,1, \ldots$ we let $\mathcal{C}_{j}$ be a collection of subsets (cells) $C$ of $\Omega_{j}$ that form a partition of $\Omega_{j}$, i.e., the sets $C$ in $\mathcal{C}_{j}$ are pairwise disjoint and their union is $\Omega_{j}$. We shall assume that $\mathcal{C}_{j}, j=0,1, \ldots$, satisfies certain properties (that we now describe) and show in the next section that these properties guarantee the existence of a multiresolution on $\Omega$. 
We assume that $\mathcal{C}_{j}$ can be partitioned into subcollections $\mathcal{C}_{j}(I, \sigma)$ for $I \subset$ $\{1, \ldots, d\}$ and $\sigma=\left(\sigma_{i}\right)_{i \in I} \subset\{-1,1\}^{|I|}$ :

$$
\mathcal{C}_{j}=\bigcup_{I, \sigma} \mathcal{C}_{j}(I, \sigma)
$$

where

$$
\mathcal{C}_{j}(I, \sigma) \cap \mathcal{C}_{j}\left(I^{\prime}, \sigma^{\prime}\right)=\varnothing \text { for }(I, \sigma) \neq\left(I^{\prime}, \sigma^{\prime}\right) .
$$

The remainder of this section will describe the further conditions we shall impose on these subcollections.

We assume that each cell $C \in \mathcal{C}_{j}(I, \sigma)$ is of the form

$$
C=\kappa+D(\kappa),
$$

with $\kappa \in \Omega_{j}$ a lattice point called the representer of $C$ and

$$
D(\kappa) \subset \operatorname{span}\left\{e_{i}: i \in I\right\} \cap 2^{-j} \mathbb{Z}^{d},
$$

with $e_{i}, i=1, \ldots, d$, the coordinate vectors in $\mathbb{R}^{d}$. We require that $\operatorname{dist}(\kappa, \partial \Omega) \leq$ $c 2^{-j}$, where $c$ does not depend on $j$, whenever $I \neq \varnothing$ and that $0 \in D(\kappa)$. The role of $\sigma$ in defining this collection will be explained subsequently.

In order to describe the other properties that we shall require of the cells $C$, we introduce the following notation. For any set $E \subset \mathbb{R}$, we define by $E^{I}$ the following subset of $\mathbb{R}^{d}$ :

$$
E^{I}=\left\{\sum_{i \in I} \lambda_{i} e_{i}: \lambda_{i} \in E, i \in I\right\}
$$

For a set $A \subset 2^{-j} \mathbb{Z}^{d}$ of lattice points and for $I \subset\{1, \ldots, d\}$, we define the spread of $A$ in the direction of $I$ by

$$
[A, I]:=\bigcup_{a \in A}\left\{a+2^{-j}[-L, L]^{I}\right\}
$$

and the spread of $A$

$$
[A]:=[A,\{1, \ldots, d\}] .
$$

If we need to indicate the dependence of the spread on level $j$ we will do this with $[A]_{j}$. However, we will simply write $[C]$ for $[C]_{j}$, if we first specify that the cell $C$ is chosen in $\mathcal{C}_{j}$.

With a given sequence $\sigma \in\{-1,1\}^{I}$ we associate the transformation $T_{\sigma}$ on $\mathbb{R}^{d}$, defined by

$$
T_{\sigma}\left(\sum_{i=1}^{d} \lambda_{i} e_{i}\right):=\sum_{i \in I} \sigma_{i} \lambda_{i} e_{i} .
$$

Now for any cell $C$ and its representer $\kappa$, we let

$$
G(C):=\left\{\kappa+2^{-j} T_{\sigma} \alpha: \alpha \in \mathbb{Z}_{+}^{d}, \alpha \leq N\right\} .
$$

Thus, $G(C)$ is a set of lattice points adjacent to $\kappa$ in the direction defined by $I$ and $\sigma$. We shall assume that the cells $C$ satisfy the following properties: 
(C1) To insure that the representer $\kappa$ of a cell $C$ is located well inside the domain $\Omega$ (which, in particular, will facilitate a simple construction of a dual system), we require that for each $C \in \mathcal{C}_{j}$,

$$
G(C) \subset C \text { and }[G(C)] \subset \Omega .
$$

(C2) This condition will ensure nestedness of the span of the dual system. Defining

$$
G_{j}:=\bigcup_{C \in \mathcal{C}_{j}} G(C), \quad j=0,1, \ldots,
$$

we require that whenever $\mu \in 2^{-j-1} \mathbb{Z}^{d}$ satisfies

$$
[\{\mu\}]_{j+1} \subseteq\left[G_{j}\right]_{j},
$$

then

$$
\mu \in G_{j+1}
$$

The next two conditions will ensure the nestedness of the multiresolution approximation spaces.

(C3) If $C \in \mathcal{C}_{j}(I, \sigma)$ and $C^{\prime} \in \mathcal{C}_{j+1}\left(I^{\prime}, \sigma^{\prime}\right)$ satisfy

$$
[C] \cap C^{\prime} \neq \varnothing
$$

then

$$
I^{\prime} \subseteq I
$$

(C4) If $C \in \mathcal{C}_{j}(I, \sigma)$ and $C^{\prime} \in \mathcal{C}_{j}\left(I^{\prime}, \sigma^{\prime}\right)$ are two cells from $\mathcal{C}_{j}$ with $C \neq C^{\prime}$ and

$$
[C, I] \cap\left[C^{\prime}, I\right] \neq \varnothing
$$

then

$$
I^{\prime} \subset I, \quad I^{\prime} \neq I .
$$

(C5) Finally, it will be important to ensure that all the basis functions have small support. There exists a constant $M$ such that

$$
\operatorname{diam}[C] \leq M 2^{-j}, \quad C \in \mathcal{C}_{j}
$$

\section{Multiresolution on $\Omega$}

We assume in this section that $\Omega$ is a bounded open set in $\mathbb{R}^{d}$ and $\phi$ is a refinable function given by the tensor product (2.2) of a univariate refinable function $\varphi$. We assume that $\varphi$ and its dual function $\tilde{\varphi}$ satisfy properties (P1)-(P4). We assume further that for each $j \geq 0, \mathcal{C}_{j}$ is a collection of cells which satisfy properties $(\mathrm{C} 1)-$ (C5). We shall construct in this section a latter of spaces $V_{j}(\Omega), j \geq 0$, which satisfy the properties of multiresolution on $\Omega$.

We begin by introducing notation that will be convenient for describing the basis for $V_{j}(\Omega)$ and establishing properties of these spaces. Let $\mathcal{L}_{j}:=2^{-j} \mathbb{Z}^{d}$ be the set of lattice points at level $j$. If $\gamma=2^{-j} k$ is a lattice point in $\mathcal{L}_{j}$, we let

$$
\phi_{\gamma}:=2^{j d / 2} \phi\left(2^{j} \cdot-k\right)
$$

be the $L_{2}$-normalized, shifted-dilate of $\phi$ corresponding to this lattice point. We need to point out that there could be some ambiguity in the above notation $\phi_{\gamma}$, since a given lattice point $\gamma$ may be in more than one of the sets $\mathcal{L}_{j}$. However, rather than revert to a more cumbersome notation such as $\phi_{\gamma, j}$, we shall simply distinguish between these basis functions by the indication $\gamma \in \mathcal{L}_{j}$ which will serve 
to indicate the dyadic level. Recall that the functions $\phi_{\gamma}, \gamma \in \mathcal{L}_{j}$, form a stable basis for $V_{j}\left(\mathbb{R}^{d}\right)$ and that all polynomials of coordinate degree $N$ are in $V_{j}\left(\mathbb{R}^{d}\right)$.

In the following, the functions $\phi_{\gamma}$ will always be viewed as being restricted to $\Omega$, unless otherwise stated, and we shall only make use of the $\phi_{\gamma}$ for $\gamma \in \Omega_{j}$.

We take an arbitrary but fixed collection $P_{0}, \ldots, P_{N}$ of univariate polynomials with $P_{k}$ of exact degree $k$. We define the multivariate polynomials

$$
P_{\alpha}\left(x_{1}, \ldots, x_{d}\right):=P_{\alpha_{1}}\left(x_{1}\right) \cdots P_{\alpha_{d}}\left(x_{d}\right),
$$

for each $\alpha \in \Lambda$ with $\Lambda:=\left\{\alpha \in \mathbb{Z}_{+}^{d}: \alpha \leq N\right\}$. Then, it follows from property (P4) that for each fixed $j \in \mathbb{N}$, the polynomials

$$
R_{\alpha, j}(x):=\sum_{\gamma \in \mathcal{L}_{j}} P_{\alpha}(\gamma) \phi_{\gamma}(x), \quad \alpha \in \Lambda,
$$

form a basis for $\Pi_{N}$. In fact, from (P4), it follows that $R_{\alpha, j}(x)$ has leading term $c x^{\alpha}$ with $c \neq 0$.

If $C \in \mathcal{C}_{j}$, say $C \in \mathcal{C}_{j}(I, \sigma)$, we let $\Lambda(C)$ be the set of all $\alpha \in \Lambda$ for which $\alpha_{j}=0$, $j \in\{1, \ldots, d\} \backslash I$. With this, we define for each $\alpha \in \Lambda(C)$,

$$
\phi_{C, \alpha}(x)=\sum_{\gamma \in C} P_{\alpha}(\gamma) \phi_{\gamma}(x), \quad x \in \Omega
$$

Each of these functions is a finite linear combination of the $\phi_{\gamma}$. We define

$$
V_{j}(\Omega):=\operatorname{span}\left\{\phi_{C, \alpha}: \alpha \in \Lambda(C), C \in \mathcal{C}_{j}\right\} .
$$

The following sections derive the properties of the spaces $V_{j}(\Omega), j \geq 0$.

\subsection{Reproduction of polynomials on $\Omega$.}

Proposition 3.1. $\Pi_{N}(\Omega) \subseteq V_{j}(\Omega)$, for all $j \geq 0$.

Proof. We fix $j \in \mathbb{N}$. Since the cells $C$ are a partition of $\Omega_{j}$, for each $\alpha \in \Lambda$, we have from (3.1),

$$
R_{\alpha, j}(x)=\sum_{\gamma \in \Omega_{j}} P_{\alpha}(\gamma) \phi_{\gamma}(x)=\sum_{C \in \mathcal{C}_{j}} \sum_{\gamma \in C} P_{\alpha}(\gamma) \phi_{\gamma}(x), \quad x \in \Omega .
$$

For a fixed $\alpha$ and $C$, we can factor $P_{\alpha}=P_{\alpha^{\prime}} P_{\alpha^{\prime \prime}}$ with $\alpha_{j}^{\prime}:=\alpha_{j}, j \in I$ and $\alpha_{j}^{\prime}:=0$, otherwise, and $\alpha^{\prime \prime}:=\alpha-\alpha^{\prime}$. Then, the polynomial $P_{\alpha^{\prime \prime}}$ is constant on $C$ and so

$$
\sum_{\gamma \in C} P_{\alpha}(\gamma) \phi_{\gamma}(x)=c \sum_{\gamma \in C} P_{\alpha^{\prime}}(\gamma) \phi_{\gamma} .
$$

Since $\alpha^{\prime}$ is in $\Lambda(C)$ the last sum is in $V_{j}(\Omega)$. It follows that each of the polynomials $R_{\alpha, j}(x), \alpha \in \Lambda$, are in $V_{j}(\Omega)$. Since these polynomials form a basis for $\Pi_{N}$ we have proved the proposition.

3.2. Nestedness. In this section, we shall show that $V_{j}(\Omega) \subset V_{j+1}(\Omega), j \geq 0$. We begin by discussing how to write a function from $V_{0}\left(\mathbb{R}^{d}\right)$ as a linear combination of the functions $\phi_{\gamma}, \gamma \in \mathcal{L}_{1}$.

From the refinement equation (2.1), we find that

$$
\begin{aligned}
\sum_{\nu \in \mathcal{L}_{0}} c_{\nu} \phi(x-\nu) & =\sum_{\nu \in \mathcal{L}_{0}} c_{\nu} \sum_{k \in \mathcal{L}_{0}} a_{k} 2^{d / 2} \phi(2 x-2 \nu-k) \\
& =\sum_{l \in \mathcal{L}_{0}}\left(\sum_{2 \nu+k=l} c_{\nu} a_{k}\right) 2^{d / 2} \phi(2 x-l)=\sum_{\gamma \in \mathcal{L}_{1}} c_{\gamma}^{\prime} \phi_{\gamma}
\end{aligned}
$$


with

$$
c_{\gamma}^{\prime}:=\sum_{\nu \in \mathcal{L}_{0}} a_{2 \gamma-2 \nu} c_{\nu}, \quad \gamma \in \mathcal{L}_{1}
$$

We recall that for $\nu=\left(\nu_{1}, \ldots, \nu_{d}\right) \in \mathcal{L}_{0}, a_{\nu}=2^{d / 2} h_{\nu_{1}} \cdots h_{\nu_{d}}$, with $h_{k}$ the univariate scaling coefficients given in (1.1).

By dilation, we obtain

$$
\sum_{\nu \in \mathcal{L}_{j}} c_{\nu} \phi_{\nu}=\sum_{\gamma \in \mathcal{L}_{j+1}} c_{\gamma}^{\prime} \phi_{\gamma}
$$

with

$$
c_{\gamma}^{\prime}:=\sum_{\nu \in \mathcal{L}_{j}} a_{2^{j+1} \gamma-2^{j+1} \nu} c_{\nu}, \quad \gamma \in \mathcal{L}_{j+1} .
$$

To show that $V_{j}(\Omega) \subset V_{j+1}(\Omega)$, it is enough to show that for each cell $C \in \mathcal{C}_{j}$, say $C \in \mathcal{C}_{j}(I, \sigma)$, and each $\alpha \in \Lambda(C)$, we have $\phi_{C, \alpha} \in V_{j+1}(\Omega)$. To this end, we let $\chi_{C}$ denote the characteristic function of $C$. Then,

$$
\phi_{C, \alpha}=\sum_{\nu \in \mathcal{L}_{j}} P_{\alpha}(\nu) \chi_{C}(\nu) \phi_{\nu}=\sum_{\gamma \in \mathcal{L}_{j+1}} P_{\alpha}^{\prime}(\gamma) \phi_{\gamma}
$$

with

$$
P_{\alpha}^{\prime}(\gamma):=\sum_{\nu \in \mathcal{L}_{j}} a_{2^{j+1} \gamma-2^{j+1} \nu} P_{\alpha}(\nu) \chi_{C}(\nu), \quad \gamma \in \mathcal{L}_{j+1} .
$$

Let $C^{\prime} \in \mathcal{C}_{j+1}\left(J, \sigma^{\prime}\right)$ be any cell in $\mathcal{C}_{j+1}$. In computing the numbers $P_{\alpha}^{\prime}(\gamma)$ for $\gamma \in C^{\prime}$, the sum in (3.4) can be restricted to those $\nu \in \mathcal{L}_{j}$ for which $[\{\nu\}] \cap C^{\prime} \neq \varnothing$ since for other values of $\nu$ the connection coefficient $a_{2^{j+1} \gamma-2^{j+1} \nu}=0$ (see properties (P1) and (P2) of $\left\{2\right.$. Here we are using our notation $[S]=[S]_{j}$ for the spread of a set $S \subset \mathcal{L}_{j}$ with respect to the level $j$. We let $\Sigma\left(C^{\prime}\right)$ be the set of all $\nu \in \mathcal{L}_{j}$ such that $[\{\nu\}] \cap C^{\prime} \neq \varnothing$. We note that by the definition of the sets $\Omega_{j}$ and $\Omega_{j+1}$, we have $\Sigma\left(C^{\prime}\right) \subset \Omega_{j}$.

To formulate the next proposition, we shall also make use the following notation. For a set $K=\left\{k_{1}, \ldots, k_{m}\right\} \subset\{1, \ldots, d\}$ with $k_{1}<k_{2}<\cdots k_{m}$, and a point $x \in \mathbb{R}^{d}$, we let $x_{K}:=\left(x_{k_{1}}, \ldots, x_{k_{m}}\right)$. Thus, $x_{K}$ is a point in $\mathbb{R}^{m}$ whose coordinates are those of $x$ corresponding to the indices in $K$. For a set $J \subset\{1, \ldots, d\}$, we let $\bar{J}:=\{1, \ldots, d\} \backslash J$.

Proposition 3.2. For each cell $C^{\prime} \in \mathcal{C}_{j+1}\left(J, \sigma^{\prime}\right)$ and each cell $C \in \mathcal{C}_{j}(I, \sigma)$ there exists a set $B \subset \mathbb{R}^{\bar{J}}$ such that

$$
\chi_{C}(\nu)=\chi_{B}(\nu \bar{J}), \quad \nu \in \Sigma\left(C^{\prime}\right) .
$$

Proof. We define $B:=\left\{\nu_{\mathcal{J}}: \nu \in \Sigma\right\}$, with $\Sigma:=\Sigma\left(C^{\prime}\right)$. To prove that (3.5) holds with this choice of $B$, it is enough tho show that whenever $\nu, \nu+2^{-j} e_{k} \in \Sigma$ with $k \in J$, then $\chi_{C}(\nu)=\chi_{C}\left(\nu+2^{-j} e_{k}\right)$. Suppose then, that we have such a $\nu$ and suppose by way of a contradiction that $\nu \in C \cap \Sigma$ but $\nu+2^{-j} e_{k} \in \widetilde{C} \cap \Sigma$ for some $k \in J$ and some cell $\widetilde{C} \in \mathcal{C}_{j}(\widetilde{I}, \tilde{\sigma})$ with $\widetilde{C} \neq C$. (A similar argument applies when $\nu+2^{-j} e_{k} \in C \cap \Sigma$ and $\nu \in \widetilde{C} \cap \Sigma$.) Since $\nu \in \Sigma$, it follows that $[\{\nu\}] \cap C^{\prime} \neq \varnothing$. Hence, $[C] \cap C^{\prime} \neq \varnothing$. From (C3), it follows that $J \subset I$. Similarly $J \subseteq \widetilde{I}$. On the other hand, since $k \in J$, it follows that $[\widetilde{C}, J] \cap C \neq \varnothing$. Hence, $[\widetilde{C}, \widetilde{I}] \cap[C, \widetilde{I}] \neq \varnothing$ 
and by $(\mathrm{C} 4), I \subset \widetilde{I}$ and $\widetilde{I} \neq I$. The same reasoning shows that $[C, I] \cap[\widetilde{C}, I] \neq \varnothing$ and therefore $I \subset I, \widetilde{I} \neq I$. This is a contradiction and proves the Proposition.

We can now provide the main result of this section.

Theorem 3.1. Let $V_{j}(\Omega)$ be defined by (3.3). Then $V_{j}(\Omega) \subset V_{j+1}(\Omega), j \in \mathbb{N}$.

Proof. Let $C \in \mathcal{C}_{j}$ and $\alpha \in \Lambda(C)$. It is enough to show that $\phi_{C, \alpha}$ is in $V_{j+1}(\Omega)$. We have

$$
\phi_{C, \alpha}(x)=\sum_{\gamma \in \Omega_{j+1}} P_{\alpha}^{\prime}(\gamma) \phi_{\gamma}(x)=\sum_{C^{\prime} \in \mathcal{C}_{j+1}} \sum_{\gamma \in C^{\prime}} P_{\alpha}^{\prime}(\gamma) \phi_{\gamma}(x), \quad x \in \Omega,
$$

with $P_{\alpha}^{\prime}$ given by (3.4). It is therefore sufficient to show that

$$
\sum_{\gamma \in C^{\prime}} P_{\alpha}^{\prime}(\gamma) \phi_{\gamma}
$$

is in $V_{j+1}(\Omega)$ for each $C^{\prime} \in \mathcal{C}_{j+1}$. Let $C^{\prime} \in \mathcal{C}_{j+1}\left(J, \sigma^{\prime}\right)$.

According to Proposition 3.2, we have for $\gamma \in C^{\prime}$,

$$
P_{\alpha}^{\prime}(\gamma)=\sum_{\nu \in \Sigma\left(C^{\prime}\right)} a_{2^{j+1} \gamma-2^{j+1} \nu} P_{\alpha}(\nu) \chi_{B}(\nu \bar{J})=\sum_{\nu \in \mathcal{L}_{j}} a_{2^{j+1} \gamma-2^{j+1} \nu} P_{\alpha}(\nu) \chi_{B}(\nu \bar{J}),
$$

where the last equality uses the fact that the connection coefficients $a_{2^{j+1} \gamma-2^{j+1} \nu}$ are zero when $\nu \notin \Sigma\left(C^{\prime}\right)$. We can write $P_{\alpha}(x)=P_{\alpha, J}\left(x_{J}\right) P_{\alpha, \bar{J}}\left(x_{\bar{J}}\right)$ with the notation $P_{\alpha, K}\left(x_{K}\right):=\Pi_{k \in K} P_{\alpha_{k}}\left(x_{k}\right)$ for any set $K \subset\{1, \ldots, d\}$. Similarly, we can factor $a_{\nu}=a_{\nu_{J}}^{J} a_{\nu_{\mathcal{J}}}^{\bar{J}}$ with $a_{\nu_{K}}^{K}:=2^{d|K| / 2} \Pi_{k \in K} h_{\nu_{k}}$. Using this in our last expression for $P_{\alpha}^{\prime}$, we find

$$
P_{\alpha}^{\prime}(\gamma)=\sum_{\mu \in B} a_{2^{j+1} \gamma_{\mathcal{J}}-2^{j+1} \mu}^{\bar{J}} P_{\alpha, \bar{J}}(\mu) \sum_{\nu J_{J}=\mu} a_{2^{j+1} \gamma_{J}-2^{j+1} \nu_{J}}^{J} P_{\alpha, J}\left(\nu_{J}\right),
$$

where we have used the fact that $\gamma_{\mathcal{J}}$ is constant for $\gamma \in C^{\prime}$.

We know from (3.1) that

$$
\sum_{\nu J_{J}=\mu} P_{\alpha, J}\left(\nu_{J}\right)\left(x_{J}\right)=R_{\alpha_{J}, j}\left(x_{J}\right) .
$$

We remark that

$$
R\left(\gamma_{J}\right):=\sum_{\nu J=\mu} a_{2^{j+1} \gamma_{J}-2^{j+1} \nu_{J}}^{J} P_{\alpha, J}\left(\nu_{J}\right)
$$

are simply the coefficients of the polynomial $R_{\alpha_{J}, j}$ when this polynomial is written as a linear combination of the $\phi_{\gamma_{J}}$, with the $\gamma_{J}$ lattice points at level $j+1$. It follows that $R$ is a polynomial of coordinate degree $N$. On the other hand,

$$
\lambda:=\sum_{\mu \in B} a_{2^{j+1} \gamma_{J^{-}} 2^{j+1} \mu}^{\bar{J}} P_{\alpha, \bar{J}}(\mu)
$$

is a constant because $\gamma_{J}$ is constant for $\gamma \in C^{\prime}$. Using these two pieces of information in (3.6), we find that

$$
P_{\alpha}^{\prime}(\gamma)=\lambda R\left(\gamma_{J}\right), \quad \gamma \in C^{\prime} .
$$

Since $R$ can be expressed as a linear combination of the polynomials $P_{\beta}$ with $\beta \in$ $\Lambda\left(C^{\prime}\right)$,

$$
\sum_{\gamma \in C^{\prime}} P_{\alpha}(\lambda) \phi_{\gamma}
$$


is a linear combination of the functions $\phi_{C^{\prime}, \beta}, \beta \in \Lambda\left(C^{\prime}\right)$. Since all of these functions are by definition in $V_{j+1}(\Omega)$, we have proven the theorem.

3.3. A new basis and a dual system. The basis functions defined in (3.2) were very convenient for proving the reproduction of polynomials in Proposition 3.1 and the nestedness of the spaces $V_{j}(\Omega)$. However, they are not so suitable for other purposes such as the construction of a simple dual system allowing to define uniformly bounded projectors onto the spaces $V_{j}(\Omega)$. Therefore, we shall now introduce another basis for $V_{j}(\Omega)$ which we shall use throughout the remainder of the paper.

For an arbitrary but fixed cell $C \in \mathcal{C}_{j}$, say $C \in \mathcal{C}_{j}(I, \sigma)$, the basis functions $\phi_{C, \alpha}$, $\alpha \in \Lambda(C)$, span a subspace $V_{C}$ of $V_{j}(\Omega)$ of dimension $(N+1)^{|I|}$. Let $\Pi_{N}(I)$ denote the space of all polynomials $P\left(x_{I}\right)$ of coordinate degree $N$. The polynomials $P_{\alpha}$, $\alpha \in \Lambda(C)$, form a basis for $\Pi_{N}(I)$. Therefore, $V_{C}$ is precisely the set of functions

$$
\sum_{\gamma \in C} P(\gamma) \phi_{\gamma}, \quad P \in \Pi_{N}(I) .
$$

We obtain other basis for $V_{C}$ by replacing the polynomials $P_{\alpha}, \alpha \in \Lambda(C)$, in the definition of the $\phi_{C, \alpha}$, by another basis for $\Pi_{N}(I)$. We next describe the basis we shall utilize for the remainder of this paper.

We recall the sets $G(C)$ of (2.12) which consists of a square array of $(N+1)^{|I|}$ lattice points from $C$. We let $P_{\nu}, \nu \in G(C)$, denote the Lagrange polynomials in $\Pi_{N}(I)$, which are defined by the interpolation conditions

$$
P_{\nu}(\gamma)=\delta_{\nu, \gamma}, \quad \gamma, \nu \in G(C) .
$$

Clearly, the polynomials $P_{\nu}, \nu \in G(C)$, form another basis for $\Pi_{N}(I)$. Hence, the functions

$$
\Phi_{\nu}:=\sum_{\gamma \in C} P_{\nu}(\gamma) \phi_{\gamma}, \quad \nu \in G(C)
$$

are another basis for $V_{C}$. We take as our new basis for $V_{j}(\Omega)$, the set of all functions

$$
\Phi_{\nu}, \quad \nu \in G_{j}
$$

where as before $G_{j}:=\bigcup_{C \in \mathcal{C}_{j}} G(C)$.

As for the $\phi_{\gamma}$, there could be some ambiguity in the above notation $\Phi_{\nu}$ since a given lattice point $\nu$ may be in more than one of the sets $G_{j}$. Again, we shall simply distinguish between these basis functions by the indication $\nu \in G_{j}$ which will serve to indicate the dyadic level.

We next define a dual basis for the $\Phi_{\nu}$. Let $\tilde{\varphi}$ be the univariate function given in (P3) which is dual to $\varphi$ and let

$$
\tilde{\phi}\left(x_{1}, \ldots, x_{d}\right):=\tilde{\varphi}\left(x_{1}\right) \cdots \tilde{\varphi}\left(x_{d}\right)
$$

be the tensor product of these functions. As with $\phi$, we define the functions

$$
\tilde{\phi}_{\gamma}(x):=2^{j d / 2} \tilde{\phi}\left(2^{j}(x-\gamma)\right), \quad \gamma \in \mathcal{L}_{j} .
$$

The functions $\phi_{\gamma}, \gamma \in \mathcal{L}_{j}$, and $\tilde{\phi}_{\gamma}, \gamma \in \mathcal{L}_{j}$, are in duality

$$
\left\langle\phi_{\gamma}, \tilde{\phi}_{\gamma^{\prime}}\right\rangle:=\int_{\mathbb{R}^{d}} \phi_{\gamma}(x) \overline{\tilde{\phi}_{\gamma^{\prime}}(x)} d x=\delta_{\gamma, \gamma^{\prime}}, \quad \gamma, \gamma^{\prime} \in \mathcal{L}_{j} .
$$


With this notation, we simply define the dual functions by

$$
\widetilde{\Phi}_{\nu}:=\tilde{\phi}_{\nu}, \quad \nu \in G(C) .
$$

Because of $(\mathrm{C} 1)$, each of the functions $\widetilde{\Phi}_{\gamma}$ is supported in $\Omega$.

The same remarks we have made earlier concerning the possible ambiguity in the notation $\Phi_{\nu}$ applies equally well here for the dual functions.

Proposition 3.3. The functions $\widetilde{\Phi}_{\gamma}, \gamma \in G_{j}$, constitute a dual system to $\Phi_{\gamma}$, $\gamma \in G_{j}$, in the sense that

$$
\left\langle\Phi_{\gamma}, \widetilde{\Phi}_{\gamma^{\prime}}\right\rangle_{\Omega}:=\int_{\Omega} \Phi_{\gamma}(x) \overline{\widetilde{\Phi}_{\gamma^{\prime}}(x)} d x=\delta_{\gamma, \gamma^{\prime}}, \quad \gamma, \gamma^{\prime} \in G_{j} .
$$

Proof. If $\gamma \in G(C)$ and $\gamma^{\prime} \in G\left(C^{\prime}\right)$ with $C \neq C^{\prime}$, then (3.11) follows from (3.9) and the fact that $G(C)$ and $G\left(C^{\prime}\right)$ are disjoint. If $\gamma, \gamma^{\prime}$ are both in the same $G(C)$, then the inner product in (3.11) equals $P_{\gamma}\left(\gamma^{\prime}\right)=\delta_{\gamma, \gamma^{\prime}}$.

Let $\widetilde{V}_{j}(\Omega)$ be the linear span of the dual functions $\widetilde{\Phi}_{\gamma}, \gamma \in G_{j}$.

Proposition 3.4. The spaces $\widetilde{V}_{j}(\Omega)$ are nested

$$
\widetilde{V}_{j}(\Omega) \subset \widetilde{V}_{j+1}(\Omega), \quad j \geq 0 .
$$

Proof. In view of 3.10 it is enough to show that each function $\tilde{\phi}_{\gamma}, \gamma \in G_{j}$, is in the space $\widetilde{V}_{j+1}(\Omega)$. We can use the refinement equation of (P3) to rewrite $\tilde{\phi}_{\gamma}$ in terms of $\tilde{\phi}_{\nu}, \nu \in \mathcal{L}_{j+1}$. The only $\tilde{\phi}_{\nu}$ which appear with a nonzero coefficient in this decomposition of $\tilde{\phi}_{\gamma}$ are those for which $\frac{1}{2}[\{\nu\}] \subset[\{\nu\}]$ with $[\cdot]:=[\cdot]_{j}$ the spread as defined in 2 . From property (C2), it follows that $\nu \in G_{j+1}$. Hence, each of the $\tilde{\phi}_{\nu}$ are in $\widetilde{V}_{j+1}(\Omega)$, as desired.

3.4. Stability of the basis. In this section, we shall show that the basis $\Phi_{\gamma}$, $\gamma \in G_{j}$, for $V_{j}(\Omega)$ is $L_{p}$-stable. We assume that the univariate functions $\varphi$ and $\tilde{\varphi}$ are in $L_{\infty}\left(\mathbb{R}^{d}\right)$.

Consider any basis function $\Phi_{\gamma}, \gamma \in G(C)$, for $V_{j}(\Omega)$. We have

$$
\Phi_{\gamma}=\sum_{\nu \in C} P_{\gamma}(\nu) \phi_{\nu}
$$

with $P_{\gamma}$ the Lagrange interpolating polynomial of $\$ 3.3$ Now, $\left|P_{\gamma}(\nu)\right| \leq c$ for $\nu \in C$, with $c$ a constant depending only on $L$ and $d$. From this, we obtain

$$
\left\|\Phi_{\gamma}\right\|_{L_{p}(\Omega)} \leq\left\|\Phi_{\gamma}\right\|_{L_{p}\left(\mathbb{R}^{d}\right)} \leq c 2^{j d(1 / 2-1 / p)}
$$

with the constant $c$ depending only on $\phi, d$ and $p$ if $p$ is close to 0 . The same conclusion holds trivially for the dual basis $\widetilde{\Phi}_{\gamma}, \gamma \in G_{j}$.

Theorem 3.2. For each $1 \leq p \leq \infty$, the bases $\left\{\Phi_{\gamma}: \gamma \in G_{j}\right\}$ are uniformly $L_{p^{-}}$ stable; i.e., there exist positive constants $c_{1}, c_{2}$, depending at most on $\phi, d$, and the closeness of $p$ to zero, such that for all $j \geq 0$ and all $\left\{\lambda_{\gamma}\right\}_{\gamma \in G_{j}}$, we have

$$
c_{1} 2^{j d(1 / 2-1 / p)}\left\|\left(\lambda_{\gamma}\right)_{\gamma \in G_{j}}\right\|_{l_{p}} \leq\left\|\sum_{\gamma \in G_{j}} \lambda_{\gamma} \Phi_{\gamma}\right\|_{L_{p}(\Omega)} \leq c_{2} 2^{j d(1 / 2-1 / p)}\left\|\left(\lambda_{\gamma}\right)_{\gamma \in G_{j}}\right\|_{l_{p}} .
$$


Similarly, the dual basis $\widetilde{\Phi}_{\gamma}, \gamma \in G_{j}$, is also $L_{p}$-stable and (3.14) holds with the $\Phi_{\gamma}$ replaced by the $\tilde{\Phi}_{\gamma}$.

Proof. We shall prove the theorem for the basis $\Phi_{\gamma}, \gamma \in G_{j}$; the same proof applies for the dual basis. We shall also assume that $p<\infty$; a similar proof applies when $p=\infty$. Let $f=\sum_{\gamma \in G_{j}} \lambda_{\gamma} \Phi_{\gamma}$. From our assumptions on the support of $\phi$ and property (C5), it follows that for each $x \in \Omega$, at most $c$ terms in the sum for $f$ are nonzero with $c$ here and later in this proof depending only on $\phi, d$, and the closeness of $p$ to zero. Therefore,

$$
|f(x)|^{p} \leq c^{p} \sum_{\gamma \in G_{j}}\left|\lambda_{\gamma}\right|^{p}\left|\Phi_{\gamma}(x)\right|^{p} .
$$

Integrating this last inequality over $\Omega$ and using (3.13), we arrive at the right inequality in (3.14).

For the lower inequality in (3.14), we use the fact that $\lambda_{\gamma}=\left\langle f, \widetilde{\Phi}_{\gamma}\right\rangle$. Hence, with $S_{\gamma}$ the support of $\widetilde{\Phi}_{\gamma}$, we have with $1 / p+1 / q=1$,

$$
\left|\lambda_{\gamma}\right|^{p} \leq c \int_{S_{\gamma}}|f|^{p}\left(\int_{S_{\gamma}}\left|\widetilde{\Phi}_{\gamma}\right|^{q}\right)^{p / q} \leq c 2^{j d(1 / 2-1 / q) p} \int_{S_{\gamma}}|f|^{p} .
$$

A point $x \in \Omega$ can appear in at most $c$ of the sets $S_{\gamma}$. Hence we can add our last inequalities over $\gamma \in G_{j}$ and arrive at the lower inequality in (3.14).

3.5. Projectors onto $V_{j}(\Omega)$ and $\widetilde{V}_{j}(\Omega)$. We can use the bases $\Phi_{\gamma}, \gamma \in G_{j}$, and $\widetilde{\Phi}_{\gamma}, \gamma \in G_{j}$, to define projectors onto the spaces $V_{j}(\Omega)$ and $\widetilde{V}_{j}(\Omega)$. In this section, we shall assume, as earlier, that $\phi$ and $\tilde{\phi}$ are in $L_{\lambda}\left(\mathbb{R}^{d}\right)$. We shall also utilize the same notation as in the previous subsection.

For each $j=0,1, \ldots$ and each $f \in L_{1}(\Omega)$, we define

$$
Q_{j} f:=\sum_{\gamma \in G_{j}}\left\langle f, \widetilde{\Phi}_{\gamma}\right\rangle_{\Omega} \Phi_{\gamma}
$$

and

$$
\widetilde{Q}_{j} f:=\sum_{\gamma \in G_{j}}\left\langle f, \Phi_{\gamma}\right\rangle_{\Omega} \widetilde{\Phi}_{\gamma}
$$

These two operators are adjoints of one another.

Theorem 3.3. For each $1 \leq p \leq \infty$, the operators $Q_{j}$ and $\widetilde{Q}_{j}$ are uniformly bounded projectors from $L_{p}(\Omega)$ onto $V_{j}(\Omega)$, respectively $\widetilde{V}_{j}(\Omega), j \geq 0$.

Proof. We shall prove the theorem for the operators $Q_{j}, j \geq 0$; the same proof applies for the adjoints $\widetilde{Q}_{j}$. We shall also assume that $p<\infty$; a similar proof applies when $p=\infty$. The same argument as given in the proof of the lower inequality in (3.14) of Theorem 3.2 shows that

$$
\left|\left\langle f, \widetilde{\Phi}_{\gamma}\right\rangle_{\Omega}\right|^{p}\left\|\Phi_{\gamma}\right\|_{p}^{p} \leq c \int_{S_{\gamma}}|f|^{p}
$$

where $c$ is a constant independent of $f$ and $j$. Hence, from Theorem 3.2, we have

$$
\left\|Q_{j} f\right\|_{p}^{p} \leq c \sum_{\gamma \in G_{j}}\left|\left\langle f, \widetilde{\Phi}_{\gamma}\right\rangle_{\Omega}\right|^{p}\left\|\Phi_{\gamma}\right\|_{p}^{p} \leq c \sum_{\gamma \in G_{j}} \int_{S_{\gamma}}|f|^{p} \leq c\|f\|_{p}^{p}
$$


because each $x \in \Phi$ appears in at most $c$ of the sets $S_{\gamma}$ (up to a modification of $c$ that does not depend on $j$ and $f$ ).

Let us point out another important fact about the operators $Q_{j}$ and $\widetilde{Q}_{j}$.

Corollary 3.1. For each $j, j^{\prime}=0,1, \ldots$ with $j^{\prime} \leq j$, we have

$$
Q_{j^{\prime}} Q_{j}=Q_{j^{\prime}}
$$

The same result holds for the adjoint projectors $\widetilde{Q}_{j}$.

Proof. For any $g \in \widetilde{V}_{j}(\Omega)$, we have (from the definition of $Q_{j}$ ) that

$$
\left\langle f-Q_{j} f, g\right\rangle_{\Omega}=0 .
$$

Since $\widetilde{V}_{j^{\prime}}(\Omega) \subset \widetilde{V}_{j}(\Omega)$, we can take $g=\widetilde{\Phi}_{\gamma}, \gamma \in G_{j^{\prime}}$. This shows that $Q_{j^{\prime}}\left(f-Q_{j} f\right)=$ 0 and proves (3.17).

Further properties of the projectors $Q_{j}$ and $\widetilde{Q}_{j}$ will be given in the following section.

\section{Approximation properties And FUnCtion spaCes}

Many applications of multiresolution rely on the approximation properties of the spaces $V_{j}$ and the characterization of various function spaces in terms of these approximation properties. Results of this type are well-known in the Euclidean case $\mathrm{Me}$. The purpose of the present section will be to generalize them to our bounded domain setting. Throughout this section, we shall assume that $\phi$ and $\tilde{\phi}$ are in $L_{\infty}\left(\mathbb{R}^{d}\right)$.

4.1. Approximation properties and Besov spaces. We shall first discuss the approximation properties of the spaces $V_{j}(\Omega)$ and in turn obtain characterizations of the Besov spaces. Let $N$ be the integer of the previous sections which indicate the polynomials contained in $V_{j}(\Omega)$. We shall assume throughout this subsection that $\phi$ is in $C^{r}\left(\mathbb{R}^{d}\right)$, and that $r \leq N+1$ with $N$ the constant of $\S \Phi 2$, 3 which indicate the degrees of polynomials contained in $V_{j}(\Omega)$.

There is a well established vehicle for proving results of the type we want. It consists of establishing two inequalities known as Jackson and Bernstein inequalities. The role of these inequalities in obtaining approximation properties and characterizing spaces is well understood. We refer the reader, for example, to the book DL, or to the papers [DS] and [DK] which treat approximation on domains as in this section.

We assume that $\Omega \subset \mathbb{R}^{d}$ is a bounded, simply connected domain (i.e. an open set) satisfying the uniform cone condition (see e.g. [A] or [M]). The uniform cone condition means that there is an open cone $K$ with vertex at the origin such that for each point $x$ on the boundary of $\Omega$ a suitable translate and rotation $K^{\prime}$ of $K$ has vertex $x$ and $K^{\prime} \cap B(x, r) \subseteq \Omega$ for some ball $B(x, r)$ centered at $x$ with radius $r$ independent of $x$.

We recall the definition of the Sobolev space $W^{r}\left(L_{p}(\Omega)\right), r=1,2, \ldots, 1 \leq p \leq$ $\infty$, which consists of all functions $f \in L_{p}(\Omega)$ whose weak derivatives of order $r$ are in $L_{p}(\Omega)$. We equip $W^{r}\left(L_{p}(\Omega)\right)$ with the usual semi-norm

$$
|f|_{W^{r}\left(L_{p}(\Omega)\right)}:=\max _{|\nu|=r}\left\|D^{\nu} f\right\|_{L_{p}(\Omega)}
$$


and the norm

$$
\|f\|_{W^{r}\left(L_{p}(\Omega)\right)}:=\|f\|_{L_{p}(\Omega)}+|f|_{W^{r}\left(L_{p}(\Omega)\right)}
$$

Let $V_{j}(\Omega), j \geq 0$, be the family of multiresolution spaces as constructed in $\S 3$ We recall the integer $N$ of that section which describes the polynomials contained in $V_{j}(\Omega)$. For a function $f \in L_{p}(\Omega)$, let

$$
E_{j}(f)_{p}:=\inf _{g \in V_{j}(\Omega)}\|f-g\|_{L_{p}(\Omega)}, \quad j \geq 0
$$

be the error in approximating $f$ by the elements of $V_{j}(\Omega)$.

Theorem 4.1. Let $r \leq N$ where $N$ is the degree of polynomial reproduction of the spaces $V_{j}(\Omega)$. For each $f \in W_{p}^{r}, 1 \leq p \leq \infty$, we have the Jackson inequality

$$
E_{j}(f) \leq\left\|f-Q_{j} f\right\|_{L_{p}(\Omega)} \leq c 2^{-r j}|f|_{W_{p}^{r}(\Omega)} .
$$

If $\phi \in C^{r}\left(\mathbb{R}^{d}\right)$, then for each $g \in V_{j}(\Omega), j \geq 0$, and each $1 \leq p \leq \infty$, we have the Bernstein inequality

$$
|g|_{W_{p}^{r}(\Omega)} \leq c 2^{j r}\|g\|_{L_{p}(\Omega)}
$$

The constants $c$ depend only on $\phi$.

Proof. The first inequality in (4.1) is obvious. We shall not prove the second inequality in detail since there are many proofs in the literature which are essentially the same. We mention only the main ingredients of the proof which are: (i) the operators $Q_{j}$ are bounded projectors onto $V_{j}(\Omega)$, (ii) polynomials of degree $N$ are locally contained in $V_{j}(\Omega)$, (iii) for each ball $B$ of radius $R$ contained in $\Omega$, there is a polynomial $P_{R}$ of degree $N$ such that

$$
\left\|f-P_{R}\right\|_{L^{p}\left(B_{R}\right)} \leq c R^{r}|f|_{W_{p}^{r}\left(B_{R}\right)}
$$

Properties (i) and (ii) were shown in $\sqrt{3}$ and property (iii) is a well-known fact on multivariate polynomial approximation (see e.g. [DS]). The proof of (4.1) from these three facts is quite straightforward. The reader may consult [DL] (Chapter 5) where the result is proved for quasi-interpolant spline operators, or [DJP] where a similar multivariate result is proved.

To prove the Bernstein inequality, let $|\nu|=r$. If $C \in \mathcal{C}_{j}$, and $\gamma \in G(C)$, then $\Phi_{\gamma}=\sum_{\mu \in C} P_{\gamma}(\mu) \phi_{\mu}$ with $P_{\gamma}$ the Lagrange polynomial of 33.3 , Note that $\left|P_{\gamma}(\mu)\right| \leq c, \mu \in C$, with $c$ an absolute constant. Since

$$
\left\|D^{\nu} \phi_{\mu}\right\|_{L_{p}\left(\mathbb{R}^{d}\right)}=2^{j r} 2^{j d(1 / 2-1 / p)}\left\|D^{\nu} \phi\right\|_{L_{p}\left(\mathbb{R}^{d}\right)} \leq c 2^{j r} 2^{j d(1 / 2-1 / p)},
$$

we have

$$
\left\|D^{\nu} \Phi_{\gamma}\right\|_{L_{p}(\Omega)} \leq 2 c^{j r} 2^{j d(1 / 2-1 / p)}, \quad|\nu|=r, \gamma \in G_{j} .
$$

Let $g=\sum_{\gamma \in G_{j}} b_{\gamma} \Phi_{\gamma}$ be an arbitrary element of $V_{j}(\Omega)$. For any point $x \in \Omega$, at most $c$ of the function $D^{\nu} \Phi_{\gamma}, \gamma \in G_{j}$, are nonzero at $x$. Hence,

$$
|g|_{W_{p}^{r}(\Omega)} \leq c 2^{j r} 2^{j d(1 / 2-1 / p)}\left\|\left(b_{\gamma}\right)_{\gamma \in G_{j}}\right\|_{l_{p}} \leq c 2^{j r}\|g\|_{L_{p}(\Omega)}
$$

where the last inequality uses the stability of the basis $\Phi_{\gamma}, \gamma \in G_{j}$ (see (3.14)).

From the Jackson and Bernstein estimates we obtain the following characterization of the Besov spaces $B_{q}^{\alpha}\left(L_{p}(\Omega)\right.$ ) (for a definition of Besov spaces and their properties see e.g. [DP] or [DS]). For the purposes of the following theorem, we define $Q_{-1}:=0$. 
Theorem 4.2. For each $1 \leq p \leq \infty$, each $0<q \leq \infty$, and each $0<\alpha<r$

$c_{1} \sum_{j \geq 0} 2^{j \alpha q}\left\|\left(Q_{j}-Q_{j-1}\right) f\right\|_{L_{p}(\Omega)}^{q} \leq\|f\|_{B_{q}^{\alpha}\left(L_{p}(\Omega)\right)}^{q} \leq c_{2} \sum_{j \geq 0} 2^{j \alpha q}\left\|\left(Q_{j}-Q_{j-1}\right) f\right\|_{L_{p}(\Omega)}^{q}$

with constants $c_{1}, c_{2}$ depending only on $\alpha, p, q$ and $\phi$.

Proof. From the Jackson and Bernstein inequality and an interpolation argument one derives the equivalence (4.3) with $\left(Q_{j+1}-Q_{j}\right) f$ replaced by $f-Q_{j} f$. Then, $f-Q_{j} f$ can be replaced by $\left(Q_{j+1}-Q_{j}\right) f$ by using discrete Hardy's inequalities. For details see, for example, [DP].

We should remark that it is also possible to characterize the Besov spaces $B_{q}^{\alpha}\left(L_{p}\right)$ when $0<p<1$ but the arguments are more involved and will not be given here (see e.g. [DP]).

The above characterization of Besov spaces is in terms of the projectors $Q_{j}$. In the case of $\mathbb{R}^{d}$, one usually goes further and replaces $\left\|\left(Q_{j}-Q_{j-1}\right) f\right\|_{L_{p}\left(\mathbb{R}^{d}\right)}$ by an equivalent expression in terms of wavelet coefficients. For example, in the univariate case, we can write

$$
\left(Q_{j}-Q_{j-1}\right) f=\sum_{k \in \mathbb{Z}} d_{j, k} \psi_{j, k},
$$

where the $\psi_{j, k}$ are the shifted dilates of the wavelet function $\psi$. Then, we can utilize the uniform stability of the wavelet basis at each dyadic level $j$. That is, there are positive constants $c_{1}, c_{2}$, depending only on $p$ and on the choice of the wavelet basis, such that for all $j \geq 0$ and all sequences $\left(\lambda_{k}\right)_{k \in \mathbb{Z}}$, we have

$$
c_{1} 2^{j(1 / 2-1 / p)}\left\|\left(\lambda_{k}\right)_{k \in \mathbb{Z}}\right\|_{l_{p}} \leq\left\|\sum_{k \in \mathbb{Z}} \lambda_{k} \psi_{j, k}\right\|_{L_{p}\left(\mathbb{R}^{d}\right)} \leq c_{2} 2^{j(1 / 2-1 / p)}\left\|\left(\lambda_{k}\right)_{k \in \mathbb{Z}}\right\|_{l_{p}} .
$$

The proof of these inequalities, which is similar to those in (3.14), uses the existence of the dual wavelet basis. Unfortunately, in our case of a domain $\Omega$, the construction of stable bases for the complement spaces $W_{j}(\Omega)$ between $V_{j}(\Omega)$ and $V_{j+1}(\Omega)$ is much more substantial and does not seem to be compatible with simple numerical computations.

We will show in the next section that it is still possible to characterize the fluctuations $\left\|\left(Q_{j}-Q_{j-1}\right) f\right\|_{p}$ in terms of sequences of coefficients. These coefficients are not obtained by the inner products of $f$ with functions from a Riesz basis but with functions from a slightly redundant frame. In return for the redundancy however, we find that the frame coefficients can be easily computed from the coefficients of $Q_{j} f$ in the basis $\left\{\Phi_{\gamma}: \gamma \in G_{j}\right\}$.

4.2. $L_{p}$-stable frames for the wavelet space $W_{j}(\Omega)$. Let $W_{j}:=W_{j}(\Omega)$ be the wavelet space consisting of all functions $\Delta_{j} f, f \in L_{p}(\Omega)$ where $\Delta_{j}:=Q_{j+1}-Q_{j}$. Then $\Delta_{j}$ is a bounded projector from $L_{p}(\Omega)$ onto $W_{j}(\Omega)$ for each $1 \leq p \leq \infty$. Correspondingly, let $\widetilde{W}_{j}:=\widetilde{W}_{j}(\Omega)$ be the dual space and $\widetilde{\Delta}_{j}$ the dual projector. Our objective in this section is to obtain an $L_{p}$-stable decomposition of the functions in $W_{j}$.

Our starting point is the biorthogonal wavelets for $\mathbb{R}^{d}$ obtained by the usual tensor product construction from the univariate functions $\varphi, \tilde{\varphi}, \psi, \tilde{\psi}$. Let $E$ be the 
set of nonzero vertices of the $d$-cube $[0,1]^{d}$, i.e. $E=\{0,1\}^{d} \backslash(0, \ldots, 0)$. For each $e \in E$, we define

$$
\Psi^{e}\left(x_{1}, \ldots, x_{d}\right):=\prod_{j=1}^{d} \eta_{e_{j}}\left(x_{j}\right),
$$

where $\eta_{0}:=\varphi$ and $\eta_{1}:=\psi$. We use a similar notation for the dual wavelets $\widetilde{\Psi}^{e}$. For each $\delta=k 2^{-j} \in \mathcal{L}_{j}$, and $e \in E$, we define for $\gamma=(\delta, e)$,

$$
\Psi_{\gamma}(x):=2^{j d / 2} \Psi^{e}\left(2^{j} x-k\right), \quad \widetilde{\Psi}_{\gamma}(x):=2^{j d / 2} \widetilde{\Psi}^{e}\left(2^{j} x-k\right) .
$$

The functions $\Psi_{\gamma}, \gamma=(\delta, e), \delta \in \mathcal{L}_{j}, e \in E$, span the wavelet space $W_{j}\left(\mathbb{R}^{d}\right)$ and the corresponding functions $\widetilde{\Psi}_{\gamma}$, span the dual spaces $\widetilde{W}_{j}\left(\mathbb{R}^{d}\right)$.

Now consider our multiresolution spaces on a domain $\Omega$. We first want to show that if $C=\{\delta\}$ is a point cell in $\mathcal{C}_{j}(\varnothing)$, then $\Psi_{\gamma}, \gamma=(\delta, e)$ is in $W_{j}$ for each $e \in E$. To see this, we rewrite $\Psi_{\gamma}$ as a linear combination of the functions $\phi_{\mu}, \mu \in \mathcal{L}_{j+1}$. Only those $\mu$ with $[\mu]_{j+1} \subseteq[\delta]_{j}$ will appear in this rewriting. From property (C3), it follows that $\{\mu\} \in \mathcal{C}_{j+1}(\varnothing)$. Hence $\Psi_{\gamma}$ is in $V_{j+1}(\Omega)$. Furthermore,

$$
\int_{\mathbb{R}^{d}} \Psi_{\gamma} \tilde{\phi}_{\mu}=0
$$

for each $\mu \in \mathcal{L}_{j}$. Since each function $\Phi_{\nu}, \nu \in G_{j}$, is a linear combination of $\phi_{\mu}$ with the support of $\phi_{\mu^{\prime}}$ contained in $\Omega$, it follows that $\left\langle\Psi_{\gamma}, \widetilde{\Phi}_{\nu}\right\rangle=0$. This shows that $Q_{j} \Psi_{\gamma}=0$ and therefore $D_{j} \Psi_{\gamma}=Q_{j+1} \Psi_{\gamma}=\Psi_{\gamma}$ (because $Q_{j+1}$ is a projector on $V_{j+1}(\Omega)$ ). Hence, we have shown that $\Psi_{\gamma} \in W_{j}(\Omega)$. A similar proof shows that the functions $\widetilde{\Psi}_{\gamma}$ are in the dual space $\widetilde{W}_{j}(\Omega)$.

We define $F_{j}=\mathcal{C}_{j}(\varnothing) \times E$ and define $W_{j}^{0}$ to be the space spanned by the $\Psi_{\gamma}$, $\gamma \in F_{j}$. Then $W_{j}^{0}$ is a closed subspace of $W_{j}(\Omega)$. Let $T_{j}$ be the projector from $L_{2}(\Omega)$ to $W_{j}^{0}$ defined by

$$
T_{j} f:=\sum_{\gamma \in F_{j}}\left\langle f, \widetilde{\Psi}_{\gamma}\right\rangle_{\Omega} \Psi_{\gamma} .
$$

Then $T_{j}$ is bounded on $L_{p}(\Omega)$, for all $1 \leq p \leq \infty$. Also, for each $f \in L_{0}(\Omega)$, we have

$$
T_{j} f=T_{j} Q_{j+1} f=T_{j} \Delta_{j} f .
$$

Indeed, the first equality in (4.5) follows because each $\widetilde{\Psi}_{\gamma}, \gamma \in F_{j}$, is in $\widetilde{V}_{j+1}(\Omega)$. While, the second equality follows from $T_{j} Q_{j}=0$ (because $\left\langle\widetilde{\Psi}_{\gamma}, \phi_{\mu}\right\rangle=0$, for all $\left.\gamma \in F_{j}, \mu \in \mathcal{L}_{j}\right)$.

We can write

$$
\Delta_{j}=T_{j}+R_{j}, \quad R_{j}:=\Delta_{j}-T_{j} .
$$

We want to obtain a representation for $R_{j}$. Let $Q_{j}^{*}$ denote the projector onto $V_{j}\left(\mathbb{R}^{d}\right)$ given by

$$
Q_{j}^{*} f=\sum_{\nu \in \mathcal{L}_{j}}\left\langle f, \tilde{\phi}_{\nu}\right\rangle \phi_{\nu}
$$

Let us consider the action of $R_{j}$ on the basis elements $\Phi_{\gamma}, \gamma \in G_{j+1}$, for $V_{j+1}(\Omega)$. We consider the following two cases:

Case 1. Whenever $[\nu]_{j} \cap[\gamma]_{j+1} \neq \varnothing, \nu \in \mathcal{L}_{j}$, then $\nu \in G_{j}(\varnothing)$. 
In this case, $Q_{j} \Phi_{\gamma}=Q_{j}^{*} \Phi_{\gamma}$ and $\Delta_{j} \Phi_{\gamma}=\Phi_{\gamma}-Q_{j}^{*} \Phi_{\gamma}$ is an element of $W_{j}\left(\mathbb{R}^{d}\right)$ and hence can be written as a linear combination of the $\Psi_{\mu}$. The only $\Psi_{\mu}$ which appear in such a decomposition are those such that the support of $\widetilde{\Psi}_{\mu}$ intersects $[\gamma]_{j+1}$. Because of our assumption in this case, all of these $\mu$ are in $F_{j}$. Hence, $\Delta_{j} \Phi_{\gamma}=T_{j} \Phi_{\gamma}$ and $R_{j} \Phi_{\gamma}=0$.

Case 2. There is a cell $C \in \mathcal{C}_{j}$ which is not a singleton and satisfies $[C]_{j} \cap[\gamma]_{j+1} \neq$ $\varnothing$.

In this case, we can write

$$
R_{j} \Phi_{j}=\Phi_{\gamma}-\sum_{\nu \in G_{j}}\left\langle\Phi_{\gamma}, \widetilde{\Phi}_{\gamma}\right\rangle_{\Omega} \Phi_{\nu}-\sum_{\tau \in F_{j}}\left\langle\Phi_{\gamma}, \widetilde{\Psi}_{\tau}\right\rangle_{\Omega} \Psi_{\tau}=\sum_{\mu \in G_{j+1}} b(\gamma, \mu) \Phi_{\mu},
$$

where the coefficients $b(\gamma, \mu)$ are obtained by rewriting each of the function $\Phi_{\nu}, \Psi_{\tau}$ (which are in $V_{j+1}(\Omega)$ ) in terms of the basis $\Phi_{\mu}, \mu \in G_{j+1}$. Note that $b(\gamma, \mu)=0$ if $|\gamma-\mu| \geq B 2^{-j}$, with a constant $B$ depending only on $L$ and $M$ in (C5). Consider, for example, the rewriting of the first sum in (4.7). If the inner product $\left\langle\Phi_{\gamma}, \widetilde{\Phi}_{\nu}\right\rangle_{\Omega}$ is not zero, then $[\gamma]_{j+1} \cap[\nu]_{j} \neq \varnothing$ and if $\Phi_{\mu}, \mu \in G_{j+1}$, appears in the rewriting of $\Phi_{\nu}$, then $[\mu]_{j+1} \subset[\nu]_{j}$. A similar argument applies to rewriting the second sum in (4.7).

Let us denote by $H_{j+1}$ the set of $\mu \in G_{j+1}$ such that $|\mu-\gamma| \leq B 2^{-j}$ for some $\gamma$ from Case 2. Then, it follows from the two above cases that for any $f \in L_{p}(\Omega)$,

$$
\begin{aligned}
R_{j} f & =R_{j} Q_{j+1} f=\sum_{\gamma \in G_{j+1}}\left\langle f, \widetilde{\Phi}_{\gamma}\right\rangle_{\Omega} R_{j} \Phi_{\gamma} \\
& =\sum_{\gamma \in G_{j+1}}\left\langle f, \widetilde{\Phi}_{\gamma}\right\rangle_{\Omega} \sum_{\mu \in G_{j+1}} b(\gamma, \mu) \Phi_{\mu}=\sum_{\mu \in H_{j+1}}\left\langle f, \Theta_{\mu}\right\rangle_{\Omega} \Phi_{\mu}
\end{aligned}
$$

with

$$
\Theta_{\mu}:=\sum_{\gamma \in G_{j+1}} b(\gamma, \mu) \widetilde{\Phi}_{\gamma}, \quad \mu \in H_{j+1} .
$$

Note that $R_{j}=R_{j} \Delta_{j}$ annihilates polynomials of degree $N$ and so $\Theta_{\mu}$ has $N+1$ vanishing moments.

We want next to observe that $H_{j+1}$ is a set contained near the boundary:

$$
\operatorname{dist}(\mu, \partial \Omega) \leq c 2^{-j}, \quad \mu \in H_{j+1},
$$

with the constant $c$ independent of $j$. Indeed, if $\gamma$ is from Case 2 above, then $\operatorname{dist}(\gamma, \partial \Omega) \leq c 2^{-j}$ because $[C]_{j} \cap[\gamma]_{j+1} \neq \varnothing$ for some nonsingleton cell (recall that, according to the assumption on their representers, nonsingleton cells have distance less than $c 2^{-j}$ from the boundary). Since any $\mu \in H_{j+1}$ satisfies $|\mu-\gamma| \leq B 2^{-j}$ for some $\gamma$ from Case 2, we have verified (4.11).

The following theorem gives the frame representation of the detail $\Delta_{j}$ and shows its $L_{p}$-stability.

Theorem 4.3. For each $f \in L_{p}(\Omega)$, we have

$$
\Delta_{j} f=\sum_{\gamma \in F_{j}}\left\langle f, \widetilde{\Psi}_{\gamma}\right\rangle_{\Omega} \Psi_{\gamma}+\sum_{\gamma \in H_{j+1}}\left\langle f, \Theta_{\gamma}\right\rangle_{\Omega} \Phi_{\gamma}
$$


and

$$
\begin{aligned}
c_{1}\left\|\Delta_{j} f\right\|_{L_{p}(\Omega)} & \leq 2^{-j d(1 / 2-1 / p)}\left(\sum_{\gamma \in F_{j}}\left|\left\langle f, \widetilde{\Psi}_{\gamma}\right\rangle_{\Omega}\right|^{p}+\sum_{\gamma \in H_{j+1}}\left|\left\langle f, \Theta_{\gamma}\right\rangle_{\Omega}\right|^{p}\right)^{1 / p} \\
& \leq c_{2}\left\|\Delta_{j}\right\|_{L_{p}(\Omega)}
\end{aligned}
$$

with the constants $c_{1}, c_{2}$ depending only on $p, \phi, \tilde{\phi}, \psi, \tilde{\psi}$, and $\Omega$.

Proof. Since $\Delta_{j}=T_{j}+R_{j}$, we have (4.12) and also

$$
\left\|\Delta_{j} f\right\|_{L_{p}(\Omega)} \leq\left\|T_{j} f\right\|_{L_{p}(\Omega)}+\left\|R_{j} f\right\|_{L_{p}(\Omega)}
$$

It is known that the wavelet basis $\Psi_{\gamma}$ is $L_{p}\left(\mathbb{R}^{d}\right)$ stable. Therefore the first term in (4.14) can be estimated by the first sum in (4.13). The second term in (4.14) can be handled in the same way using the stability of the basis $\Phi_{\gamma}, \gamma \in G_{j+1}$. We have therefore proved the left inequality in (4.13).

Conversely, (4.5) implies $T_{j} f=T_{j} \Delta_{j} f$ and thus $\left\|T_{j} f\right\|_{L_{p}(\Omega)} \leq c\left\|\Delta_{j} f\right\|_{L_{p}(\Omega)}$. Likewise, we have $R_{j} f=R_{j} \Delta_{j} f$ and therefore $\left\|R_{j} f\right\|_{L_{p}(\Omega)} \leq c\left\|\Delta_{j} f\right\|_{L_{p}(\Omega)}$. Therefore, the right inequality in (4.13) follows again from the stability of the two bases.

4.3. Characterization of $L_{p}$ spaces. Let $f \in L_{p}(\Omega)$. From the results of $\$ 4.1$ it follows that

$$
\lim _{j \rightarrow \infty}\left\|Q_{j} f-f\right\|_{p}=0 .
$$

(Indeed, this is the case for functions in a Besov space $B_{q}^{\alpha}\left(L_{p}(\Omega)\right)$ and these functions are dense in $L_{p}(\Omega)$.) Using the representation of $\Delta_{j} f$ given in the last subsection, we have

$$
f=\sum_{\gamma \in G_{0}}\left\langle f, \widetilde{\Phi}_{\gamma}\right\rangle_{\Omega} \Phi_{\gamma}+\sum_{j=0}^{\infty}\left(\sum_{\gamma \in F_{j}}\left\langle f, \widetilde{\Psi}_{\gamma}\right\rangle_{\Omega} \Psi_{\gamma}+\sum_{\gamma \in H_{j+1}}\left\langle f, \Theta_{\gamma}\right\rangle_{\Omega} \Phi_{\gamma}\right),
$$

where the series converges in $L_{p}(\Omega)$. The purpose of the present section is to go further and show that the above series is unconditionally convergent and thereby obtain a characterization of the $L_{p}$-norm of $f$ by certain square functions.

In order to prove that the series (4.15) is unconditionally convergent, we have to show that the operators formally defined by

$$
\begin{aligned}
T_{\omega} f= & \sum_{\gamma \in G_{0}} \omega_{\gamma}\left\langle f, \widetilde{\Phi}_{\gamma}\right\rangle_{\Omega} \Phi_{\gamma} \\
& +\sum_{j=0}^{\infty}\left(\sum_{\gamma \in F_{j}} \omega_{\gamma}\left\langle f, \widetilde{\Psi}_{\gamma}\right\rangle_{\Omega} \Psi_{\gamma}+\sum_{\gamma \in H_{j+1}} \omega_{\gamma}\left\langle f, \Theta_{\gamma}\right\rangle_{\Omega} \Phi_{\gamma}\right),
\end{aligned}
$$

are uniformly bounded on $L_{p}(\Omega)$, independently of the choice of the sequence $\omega_{\gamma}=$ \pm 1 .

Remark 4.1. We want first to observe that the operator $T_{\omega}$ naturally extends to an operator $U_{\omega}$ defined for functions in $L_{p}\left(\mathbb{R}^{d}\right)$. Indeed, the inner products in (4.16) are all defined for any $f \in L_{p}\left(\mathbb{R}^{d}\right)$ and, in view of (3.7), the functions $\Phi_{\gamma}, \gamma \in G_{j}$, 
have a natural extension on the whole of $\mathbb{R}^{d}$. Therefore, we can define $U_{\omega}$ by the corresponding extension of the right-hand side in (4.16). It is clear that

$$
\left\|T_{\omega}\right\|_{L_{p}(\Omega)} \leq\left\|U_{\omega}\right\|_{L_{p}\left(\mathbb{R}^{d}\right)}
$$

since $T_{\omega} f=U_{\omega} g$, where $g=f$ on $\Omega, g=0$ elsewhere. We shall now focus on proving the uniform boundedness in $L_{p}\left(\mathbb{R}^{d}\right)$ of the operators $U_{\omega}$.

To prove that the operators $U_{\omega}$ are $L_{p}$-bounded independent of $\omega$, we shall use the theory of Calderon-Zygmund operators and in particular, the celebrated " $T(1)$ theorem" of G. David and J. L. Journé. For the reader unfamiliar with this theory, we remark that we could prove the $L_{p}$ boundedness of these operators directly but at the price of numerous technicalities. In particular, a method was developed by W. Dahmen in [D2] to prove the $L^{2}$-stability in a very general setting: one essentially requires a Jackson and Bernstein estimate for $L_{2}$-Sobolev spaces, for both the spaces $V_{j}$ and the dual spaces $\widetilde{V}_{j}$. Note that in our situation, the dual spaces $\widetilde{V}_{j}$ do not even contain constant functions and thus a Jackson estimate will only be possible for Sobolev spaces of small index.

The application of the Calderon-Zygmund operator theory to establishing Littlewood-Paley theorems of the type we shall obtain is well documented. In particular, our development is very close to that given for the usual wavelet decompositions on $\mathbb{R}^{d}$ in the second volume of $\mathrm{Me}$. For this reason, we shall be brief and only indicate the main steps and the variances in our case with the usual case of wavelet decompositions.

By definition, a Calderon-Zygmund operator is an $L^{2}\left(\mathbb{R}^{d}\right)$-bounded integral operator whose kernel $K(x, y), x, y \in \mathbb{R}^{d}$, satisfies the following estimates

$$
\begin{array}{cc}
|K(x, y)| \leq c|x-y|^{-d}, & \\
\left|K(x, y)-K\left(x^{\prime}, y\right)\right| \leq c\left|x-x^{\prime}\right|^{s}|x-y|^{-d-s}, & \left|x-x^{\prime}\right| \leq|x-y| / 2, \\
\left|K(x, y)-K\left(x, y^{\prime}\right)\right| \leq c\left|y-y^{\prime}\right|^{s}|x-y|^{-d-s}, & \left|y-y^{\prime}\right| \leq|x-y| / 2,
\end{array}
$$

for some $s>0$.

We will use two important results of the theory of Calderon-Zygmund operators.

Theorem 4.4. A Calderon-Zygmund operator is bounded on $L_{p}\left(\mathbb{R}^{d}\right), 1<p<\infty$, and its norm on these spaces only depends on its $L_{2}$ norm and the values of the constants in 4.17) 4.19).

The second result is the $T(1)$ theorem which gives a necessary and sufficient condition for the $L_{2}$ boundedness of an operator that satisfies (4.17)-(4.19). Let $T$ be a continuous linear operator from the space of test functions $\mathcal{D}\left(\mathbb{R}^{d}\right)$ to the distribution space $\mathcal{D}^{\prime}\left(\mathbb{R}^{d}\right)$ such that its distribution kernel satisfies (4.17)-(4.19). We say that $T$ is weakly continuous on $L_{2}\left(\mathbb{R}^{d}\right)$ if and only if one has

$$
|T\langle T f, g\rangle| \leq c R^{d}\left(\|f\|_{\infty}+R\|\nabla f\|_{\infty}\right)\left(\|g\|_{\infty}+R\|\nabla g\|_{\infty}\right),
$$

for all $f, g \in \mathcal{D}\left(\mathbb{R}^{d}\right)$ whose support is contained in a ball of radius $R$.

We also define the space $B M O\left(\mathbb{R}^{d}\right)$ (bounded mean oscillation) as the set of functions in $L_{2}^{\text {loc }}$ such that

$$
\|f\|_{B M O}:=\sup _{Q}\left(|Q|^{-1} \int_{Q}\left|f(x)-m_{Q} f\right|^{2} d x\right)^{1 / 2}<\infty,
$$


where the supremum is taken over all cubes $Q$ of $\mathbb{R}^{d}$ and where $m_{Q} f=$ $|Q|^{-1} \int_{Q} f(x) d x$.

Theorem 4.5. Let $T$ be an integral operator that is weakly continuous on $L_{2}\left(\mathbb{R}^{d}\right)$ and satisfies the conditions (4.17)-(4.19). Then $T$ defines a bounded operator on $L_{2}\left(\mathbb{R}^{d}\right)$ if and only if $T(1), T^{*}(1) \in B M O\left(\mathbb{R}^{d}\right)$. Moreover the norm of $T$ on $L_{2}\left(\mathbb{R}^{d}\right)$ depends only on the constants in (4.17) (4.20) and on the norms of $T(1), T^{*}(1)$ in $B M O$.

Note that in general $T(1)$ and $T^{*}(1)$ do not make sense a priori, but they can be well defined by a limiting process in the distribution sense. In our particular case, their definition will not require this process, because of the form of the operator $U_{\omega}$.

We now turn to the operators $U_{\omega}$, defined by the kernels

$$
\begin{aligned}
K_{\omega}(x, y)= & \sum_{\gamma \in G_{0}} \omega_{\gamma} \widetilde{\Phi}_{\gamma}(y) \Phi_{\gamma}(x) \\
& +\sum_{j=0}^{\infty}\left[\sum_{\gamma \in F_{j}} \omega_{\gamma} \widetilde{\Psi}_{\gamma}(y) \Psi_{\gamma}(x)+\sum_{\gamma \in H_{j+1}} \omega_{\gamma} \Theta_{\gamma}(y) \Phi_{\gamma}(x)\right],
\end{aligned}
$$

where the functions $\Phi_{\gamma}$ have been extended outside of $\Omega$, according to 4.1).

In order to ensure the conditions (4.17)-4.19), we shall assume that the functions $\varphi, \tilde{\varphi}$ are in $B_{\infty}^{s}\left(L_{\infty}\right)$ for some $s>0$. Under this assumption, it is immediate to check (4.17)-(4.19), using the following properties of the functions that make up the kernels

- The diameter of the supports of $\Phi_{\alpha}, \Theta_{\beta}, \Psi_{\gamma}$ and $\widetilde{\Psi}_{\gamma},(\alpha, \beta, \gamma) \in G_{j} \times H_{j+1} \times F_{j}$ are less than $c 2^{-j}$.

- For a fixed $j$, each $x \in \mathbb{R}^{d}$ is contained in at most $m$ of the supports of $\Phi_{\alpha}, \Theta_{\beta}, \Psi_{\gamma}$ and $\widetilde{\Psi}_{\gamma},(\alpha, \beta, \gamma) \in G_{j} \times H_{j+1} \times F_{j}$, where $m$ is independent of $j$.

- The $L_{\infty}$ norm of $\Phi_{\alpha}, \Theta_{\beta}, \Psi_{\gamma}, \widetilde{\Psi}_{\gamma},(\alpha, \beta, \gamma) \in G_{j} \times H_{j+1} \times F_{j}$, satisfy uniformly the estimate $|\eta(x)-\eta(y)| \leq c 2^{d j / 2} 2^{j s}|x-y|^{s}$, where $c$ does not depend on $j$.

It is also clear that the constants in (4.17) -4.19) will be independent of $\omega$.

Theorem 4.6. The operators $U_{\omega}$ are weakly continuous on $L_{2}$ with a uniform constant in (4.20). Moreover, $U_{\omega}(1)$ and $U_{\omega}^{*}(1)$ are uniformly bounded in BMO. As a consequence, the operators $T_{\omega}$ are uniformly bounded on $L_{p}(\Omega), 1<p<\infty$.

Proof. From the characterization of Besov spaces by the multiscale approximation (see \$4.1), we know that the operators $U_{\omega}$ are well defined on spaces of sufficiently smooth functions.

Next, we want to bound the coefficients in the representation (4.16) of $U_{\omega}$. Suppose that $f \in C^{1}$ is supported on a ball $B_{R}$ of radius $R$ and $\|f\|_{\infty}+\|\nabla f\|_{\infty}<\infty$. Then, for each $\gamma \in F_{j}$, we define $a_{\gamma}$ to be the mean value of $f$ on the support of $\widetilde{\Psi}_{\gamma}$, if this support is contained in $B_{R}$, and we set $a_{\gamma}=0$ in the opposite case. It follows that

$$
\begin{aligned}
\left|\left\langle f, \widetilde{\Psi}_{\gamma}\right\rangle\right| & =\left|\left\langle f-a_{\gamma}, \widetilde{\Psi}_{\gamma}\right\rangle\right| \leq c\|\nabla f\|_{\infty} \min \left(R, 2^{-j}\right) \int_{B_{R}}\left|\widetilde{\Psi}_{\gamma}\right| \\
& \leq c 2^{j d / 2}\|\nabla f\|_{\infty}\left(\min \left(R, 2^{-j}\right)\right)^{d+1}
\end{aligned}
$$


where we have used the fact that $\widetilde{\Psi}_{\gamma}$ has mean value 0 , support in a set of measure $m \leq c 2^{-j d}$ and $\left\|\widetilde{\Phi}_{\gamma}\right\|_{\infty} \leq c 2^{j d / 2}$. Exactly the same reasoning shows that for each $\gamma \in H_{j+1}$,

$$
\left|\left\langle f, \Theta_{\gamma}\right\rangle\right| \leq c 2^{j d / 2}\|\nabla f\|_{\infty}\left(\min \left(R, 2^{-j}\right)\right)^{d+1} .
$$

On the other hand, for $\gamma \in \mathcal{L}_{0}$, we have

$$
\left|\left\langle f, \Phi_{\gamma}\right\rangle\right| \leq c\left\|\varphi^{N, d}\right\|_{\infty}
$$

Using these estimates to estimate the sum of the absolute values of the series appearing in the definition (4.16) of $U_{\omega}$, we obtain

$$
\left\|U_{\omega} f\right\|_{\infty} \leq c\left(\|f\|_{\infty}+R\|\nabla f\|_{\infty}\right)
$$

where the constant $c$ is independent of $\omega$. From this, the weak continuity (4.20) of $U_{\omega}$ follows immediately.

In order to prove the uniform $L_{p}$ boundedness of our family of operators, it thus remains to show that $U_{\omega}(1)$ and $U_{\omega}^{*}(1)$ are uniformly bounded in $B M O$. We shall prove here that these functions are uniformly bounded in the $L_{\infty}$ norm which clearly dominates the $B M O$ norm.

We first treat the case of the operators $U_{\omega}$. Since the functions $\Psi_{\gamma}$ and $\Theta_{\gamma}$ have zero integral, we have

$$
\left|U_{\omega}(1)(x)\right| \leq \sup _{\gamma \in G_{0}}\left|\int \widetilde{\Phi}_{\gamma}\right| \sum_{\gamma \in G_{0}}\left|\Phi_{\gamma}(x)\right| .
$$

Since the functions $\Phi_{\gamma}, \gamma \in G_{0}$, are uniformly bounded and for each $x$ there are at most $c$ of these functions which are nonzero at $x$, we have that $\left\|U_{\omega}(1)\right\|_{\infty} \leq c$ with the constant $c$ independent of $\omega$.

The case of the operators $U_{\omega}^{*}$ is slightly more delicate since one needs to analyze the contribution of the nonzero term

$$
\Sigma_{\omega}:=\sum_{j \geq 0} \sum_{\gamma \in H_{j+1}}\left\langle 1, \Phi_{\gamma}\right\rangle \Theta_{\gamma}
$$

We first note the estimate

$$
\max _{\gamma \in H_{j+1}}\left|\left\langle 1, \Phi_{\gamma}\right\rangle\right| \leq \max _{\gamma \in H_{j+1}} \int\left|\Phi_{\gamma}\right| \leq c 2^{-j d / 2},
$$

where $c$ does not depend on $j$. Thus,

$$
\left|\Sigma_{\omega}(x)\right| \leq c \sum_{j \geq 0} 2^{-j d / 2} \sum_{\gamma \in H_{j+1}}\left|\Theta_{\gamma}(x)\right| .
$$

Since each $x \in \mathbb{R}^{d}$ lies in the support of at most $m$ functions $\Theta_{\gamma}, \gamma \in H_{j+1}$ where $m$ is independent of $j$, we can replace (4.25) by

$$
\left|\Sigma_{\omega}(x)\right| \leq c \sum_{j \geq 0} 2^{-j d / 2} \max _{\gamma \in H_{j+1}}\left|\Theta_{\gamma}(x)\right| .
$$

Consider now $x \in \mathbb{R}^{d}$ and $j_{x} \in \mathbb{Z}$ such that $2^{-j_{x}-1}<d(x, \partial \Omega) \leq 2^{-j_{x}}$. From (4.11), we know that the supports of the $\Theta_{\gamma}, \gamma \in H_{j+1}$, are contained in $\left\{x ; d(x, \partial \Omega) \leq c_{0} 2^{-j}\right\}$, where $c_{0}$ is a fixed constant. Therefore, for a fixed but suitably large constant $a \geq 1$, we have $\Theta_{\gamma}(x)=0$, whenever $\gamma \in H_{j+1}$ and $j \geq a+j_{x}$ 
and consequently,

$$
\left|\Sigma_{\omega}(x)\right| \leq c \sum_{0 \leq j \leq a+j_{x}} 2^{-j d / 2} \max _{\gamma \in H_{j+1}}\left|\Theta_{\gamma}(x)\right| .
$$

From our assumption that $\phi$ is in $B_{\infty}^{s}\left(L_{\infty}\left(\mathbb{R}^{d}\right)\right)$, it follows that

$$
\left|\Theta_{\gamma}\right|_{B_{\infty}^{s}\left(L_{\infty}\left(\mathbb{R}^{d}\right)\right)} \leq 2^{j s} 2^{j d / 2}
$$

whenever $\gamma \in H_{j+1}$. Also, $\Theta_{\gamma}$ vanishes on $\partial \Omega$. Thus, if we choose $y \in \partial \Omega$ as the closest point from $\partial \Omega$ to $x$, we obtain

$$
2^{-j d / 2}\left|\Theta_{\gamma}(x)\right| \leq c^{j s}|x-y|^{s} \leq c 2^{j s} 2^{-j_{x} s},
$$

where $c$ is independent of $x, j$, and $\gamma$.

We use (4.28) in (4.27) to find

$$
\left|\Sigma_{\omega}(x)\right| \leq c \sum_{0 \leq j \leq a+j_{x}} 2^{j s} 2^{-j_{x} s} \leq c,
$$

with $c$ independent of $x \in \mathbb{R}^{d}$. This proves that $U_{\omega}^{*}(1)$ is in $L_{\infty}$ with a bound independent of $\omega$ and therefore completes the proof of our theorem.

From the uniform $L_{p}$ boundedness of the operators, one can apply the same arguments as in the Euclidean case (see $\left[\mathrm{Me}\right.$ ) in order to prove that the $L_{p}(\Omega)$ norm of a function $f$ is equivalent to the $L_{p}(\Omega)$ norm of the following 'square functions'

$$
\begin{aligned}
\sigma_{1}(f)= & \left(\sum_{\gamma \in G_{0}}\left|\left\langle f, \widetilde{\Omega}_{\gamma}\right\rangle_{\Omega} \Phi_{\gamma}\right|^{2}\right. \\
& \left.+\sum_{j=0}^{+\infty}\left(\sum_{\gamma \in F_{j}}\left|\left\langle f, \widetilde{\Psi}_{\gamma}\right\rangle_{\Omega} \Psi_{\gamma}\right|^{2}+\sum_{\gamma \in H_{j+1}}\left|\left\langle f, \Theta_{\gamma}\right\rangle_{\Omega} \Phi_{\gamma}\right|^{2}\right)\right)^{1 / 2},
\end{aligned}
$$

or

$$
\begin{aligned}
\sigma_{1}(f)= & \left(\sum_{\gamma \in G_{0}}\left|\left\langle f, \widetilde{\Omega}_{\gamma}\right\rangle_{\Omega}\right|^{2} \chi_{\gamma}\right. \\
& \left.\quad+\sum_{j=0}^{+\infty} 2^{d j}\left(\sum_{\gamma \in F_{j}}\left|\left\langle f, \widetilde{\Psi}_{\gamma}\right\rangle_{\Omega}\right|^{2} \chi_{\gamma}+\sum_{\gamma \in H_{j+1}}\left|\left\langle f, \Theta_{\gamma}\right\rangle_{\Omega}\right|^{2} \chi_{\gamma}\right)\right)^{1 / 2},
\end{aligned}
$$

where $\chi_{\gamma}$ is simply the characteristic function of the dyadic cube with lower vertex $\gamma$ and sidelength $2^{-j}$ if $\gamma \in F_{j} \cup G_{j} \cup H_{j+1}$.

In the case $p=2$, we derive from this the stability result

$$
\|f\|_{L_{2}(\Omega)}^{2} \sim \sum_{\gamma \in G_{0}}\left|\left\langle f, \widetilde{\Phi}_{\gamma}\right\rangle_{\Omega}\right|^{2}+\sum_{j=0}^{+\infty}\left(\sum_{\gamma \in F_{j}}\left|\left\langle f, \widetilde{\Psi}_{\gamma}\right\rangle_{\Omega}\right|^{2}+\sum_{\gamma \in H_{j+1}}\left|\left\langle f, \Theta_{\gamma}\right\rangle_{\Omega}\right|^{2}\right) .
$$

Finally, we remark that the characterization of function spaces in $\Omega$ by our multiscale decomposition yields a simple extension theorem. 
Theorem 4.7. The operator $U_{\omega}$ with all $\omega_{\gamma}:=1$ provides an extension operator for functions on $\Omega$ (extending them to $\mathbb{R}^{d}$ ). This extension operator is bounded from $L_{p}(\Omega)$ to $L_{p}\left(\mathbb{R}^{d}\right)$ for each $1<p<\infty$ and is also bounded from $B_{q}^{\alpha}\left(L_{p}(\Omega)\right)$ to $B_{q}^{\alpha}\left(L_{p}\left(\mathbb{R}^{d}\right)\right)$ for each $1 \leq p \leq \infty, 0<q \leq \infty$ and $0<\alpha<r$.

This extension can be viewed as a numerically implementable version of the operator constructed by Stein in [S], since it operates in domain with the same minimal smoothness assumptions on the boundary, namely Lipschitz behaviour, as we shall now see in the bidimensional case.

\section{Domains $\Omega$ IN $\mathbb{R}^{2}$}

In this section, we shall give a class of domains $\Omega$ in $\mathbb{R}^{2}$ and construct for these domains a partition of $\Omega_{j}$ into a collection $\mathcal{C}_{j}$ of cells which satisfy the conditions $(\mathrm{C} 1-5)$ of $\oint_{2}$. Thus, for these domains, the construction of the multiresolution spaces $V_{j}(\Omega)$ and all the ensuant properties given in $\$ 3$ and $\S 4$ hold. We begin by describing the conditions we shall assume about the domain $\Omega$.

We assume that $\Omega$ is a bounded, simply connected domain (i.e. an open set) satisfying the uniform cone condition. We recall that the uniform cone condition means that there is an open cone $K$ with vertex at the origin such that for each point $x$ on the boundary of $\Omega$ a suitable translate and rotation $K^{\prime}$ of $K$ has vertex $x$ and $K^{\prime} \cap B(x, r) \subseteq \Omega$ for some ball $B(x, r)$ centered at $x$ with radius $r$.

We assume that the exterior domain $\Omega^{c}$, by which we mean the interior of the complement of $\Omega$, also satisfies the uniform cone condition. We shall use $(x, y)$ (instead of $\left.\left(x_{1}, x_{2}\right)\right)$ to denote the points in $\mathbb{R}^{2}$ in this section. We also assume that the boundary of $\Omega$ is a simple closed curve $\Gamma$ which is the union of curves $\Gamma_{k}, k=$ $1, \ldots, m$, with the following properties. $\Gamma_{k}$ has endpoints $p_{k-1}$ and $p_{k}:=\left(x_{k}, y_{k}\right)$, when traversed in a clockwise direction. For $k$ even, $\Gamma_{k}$ can be parameterized by $\left(x, \gamma_{k}(x)\right), x \in I_{k}$, with $I_{k}$ an interval with endpoints $x_{k-1}$ and $x_{k}$ and $\gamma_{k}$ is in $\operatorname{Lip}_{M} 1$. Recall that $\operatorname{Lip}_{M} 1$ is the set of all continuous univariate functions $g$ which satisfy $\left\|g^{\prime}\right\|_{\infty} \leq M$. We shall assume (without loss of generality) that $M \geq 1$. When $k$ is odd, $\Gamma_{k}$ can be parameterized by $\left(\gamma_{k}(y), y\right), y \in I_{k}$, with $I_{k}$ an interval with endpoints $y_{k-1}$ and $y_{k}$ and $\gamma_{k}$ is in $\operatorname{Lip}_{M} 1$. The points $p_{m}$ and $p_{0}$ coincide.

For each $k$, we denote by $\nu_{k}$ a vector $(0, \pm 1)$ or $( \pm 1,0)$ which indicates the direction exterior to $\Omega$ from $\Gamma_{k}$. For example, if $k$ is even, then $\nu_{k}=(0, \pm 1)$, and the points $\left(x, \gamma_{k}(x)\right)+h \nu_{k}$ are not in $\Omega$ provided $x$ is in the interior of $I_{k}$ and $h$ is sufficiently small (depending on $x$ ); correspondingly, the points $\left(x, \gamma_{k}(x)\right)-h \nu_{k}$ are in $\Omega$ provided $h$ is sufficiently small. A similar definition with $\nu_{k}=( \pm 1,0)$ applies when $k$ is odd.

We now derive a few useful properties of $\Omega$ and $\Gamma$. In what follows, we shall denote by $Q(x, h)$ the square centered at $x$ with sidelength $2 h$ (all squares in this paper have sides parallel to the coordinate axis). For any set $A$, we denote by $Q(A, h)$ the neighborhood of $A$ which consists of the union of the squares $Q(x, h)$, $x \in A$.

We can assume that the cone $K$ also satisfies the cone condition for $\Omega^{c}$. For each $k=0, \ldots, m$ and each $p_{k}$, there is a cone $K\left(p_{k}\right)$ which is a translate and rotation of $K$ so that $p_{k}$ is the vertex of $K\left(p_{k}\right)$ and $K\left(p_{k}\right) \cap Q\left(p_{k}, h\right)$ is contained in $\Omega$ for all $h$ sufficiently small, say $h \leq h_{0}$. Similarly, there is a cone $\widetilde{K}\left(p_{k}\right)$ with this same property relative to $\Omega^{c}$. 
We want to make a distinction between "inside" and "outside" corners for the points $p_{k}, k=0, \ldots, m$, and to make some further stipulations on the cones $K\left(p_{k}\right)$ and $\widetilde{K}\left(p_{k}\right)$. We say $p_{k}$ is an outside corner for $\Omega$ if $\operatorname{det}\left(\nu_{k}^{T}, \nu_{k+1}^{T}\right)<0$; similarly, we say $p_{k}$ is an inside corner for $\Omega$ if $\operatorname{det}\left(\nu_{k}^{T}, \nu_{j+1}^{T}\right)>0$. Because of the Lipschitz condition on $\Gamma$, we can choose $K$ so that whenever $p_{k}, k=0, \ldots, m$, is an outside corner, then $K\left(p_{k}\right)$ is locally contained in the sector $\left\{p_{k}-\alpha \nu_{k}-\beta \nu_{k+1}: \alpha, \beta>0\right\}$. Here locally means that some ball $B\left(p_{k}, r\right)$ intersected with $K\left(p_{k}\right)$ is contained in the specified sector. For an outside corner, the sector $\left\{p_{k}+\alpha \nu_{k}+\beta \nu_{k+1}: \alpha, \beta>0\right\}$ is locally contained in $\Omega^{c}$ and $\widetilde{K}\left(p_{k}\right)$ is contained in this sector. For an inside corner, the situation reverses: the sector $\left\{p_{k}-\alpha \nu_{k}-\beta \nu_{k+1}: \alpha, \beta>0\right\}$ is contained in $\Omega$ and $K\left(p_{k}\right)$ is contained in this sector, but for $\Omega^{c}$, we can only say that the sector $\left\{p_{k}+\alpha \nu_{k}+\beta \nu_{k+1}: \alpha, \beta>0\right\}$ contains the cone $\widetilde{K}\left(p_{k}\right)$.

In what follows $\lambda$ will denote a constant which will be required to be sufficiently large that certain conditions are valid. As we proceed, we may impose further conditions on $\lambda$ which are always satisfied if $\lambda$ is large enough. After we have introduced all our restrictions on $\lambda$, we will fix $\lambda$. In the choice of $\lambda$ and other constants in this section, we make no attempt to choose the best constants rather to choose these constants so that the desired properties are most apparent.

We next define certain "corner sets" associated with the $p_{k}, k=0, \ldots, m$. If $p_{k}$ is an outside corner for $\Omega$, we let $L_{k}=\left\{p_{k}-t\left(\alpha_{k} \nu_{k}+\beta_{k} \nu_{k+1}\right): t>0\right\}$ be the ray emanating from $p_{k}$ which is the middle ray of $K\left(p_{k}\right)$. It follows that $\alpha_{k}, \beta_{k}>0$. For each $h>0$ and for our $\lambda$, let $q_{k}(h):=p_{k}-\lambda h\left(\alpha_{k} \nu_{k}+\beta_{k} \nu_{k+1}\right)$ which is a point on $L_{k}$. The sector $\left\{q_{k}(h)+\alpha \nu_{k}+\beta \nu_{k+1}: \alpha, \beta \geq 0\right\}$ contains a portion of $\Gamma_{k}$ and $\Gamma_{k+1}$. We define $\mathcal{S}_{\mathbf{k}}$ to be the set of points on $\Gamma_{k} \cup \Gamma_{k+1}$ which are in this sector. If $k$ is even, we define $Q_{k}(h)$ to be the smallest square with vertex $q_{k}(h)$ which contains all of the line segments with vertices $\left(x, \gamma_{k}(x)\right)$ and $\left(x, \gamma_{k}(x)\right)+\lambda h \nu_{k}$ for each $\left(x, \gamma_{k}(x)\right) \in \mathcal{S}_{\mathbf{k}}$ and the line segments with vertices $\left(\gamma_{k+1}(y), y\right)$ and $\left(\gamma_{k+1}(y), y\right)+\lambda h \nu_{k+1}$ for each $\left(\gamma_{k+1}(y), y\right) \in \mathcal{S}_{\mathbf{k}}$. We define $Q_{k}(h)$ in a similar way if $k$ is odd. In the case that $p_{k}$ is an inside corner, we construct the square $Q_{k}(h)$ relative to $\Omega^{c}$. That is, we define $q_{k}(h):=p_{k}+\lambda h\left(\tilde{\alpha}_{k} \nu_{k}+\tilde{\beta}_{k} \nu_{k+1}\right)$ which is a point on the middle ray $\widetilde{L}_{k}$ of the cone $\widetilde{K}\left(p_{k}\right)$. Then, $\mathcal{S}_{\mathbf{k}}:=\left\{q_{k}(h)-\alpha \nu_{k}-\beta \nu_{k+1}: \alpha, \beta \geq 0\right\} \cap\left(\Gamma_{k} \cup \Gamma_{k+1}\right)$ and $Q_{k}(h)$ is the smallest square with vertex $q_{k}(h)$ which contains all line segments vertices $\left(x, \gamma_{k}(x)\right)$ and $\left(x, \gamma_{k}(x)\right)-\lambda h \nu_{k}$ for each $\left(x, \gamma_{k}(x)\right) \in \mathcal{S}_{\mathbf{k}}$ and the line segments with vertices $\left(\gamma_{k+1}(y), y\right)$ and $\left(\gamma_{k+1}(y), y\right)-\lambda h \nu_{k+1}$ for each $\left(\gamma_{k+1}(y), y\right) \in \mathcal{S}_{\mathbf{k}}$.

If $h$ is sufficiently small, the squares $Q_{k}(h)$ are pairwise disjoint. We consider only such $h$ in what follows. It is also clear that the sidelength of $Q_{k}(h)$ does not exceed $c \lambda M h$ with $c$ a constant (depending only on the $\alpha_{k}, \beta_{k}, \tilde{\alpha}_{k}, \tilde{\beta}_{k}$ ) and $M$ the Lipschitz constant for $\Gamma$.

Proposition 5.1. If $\lambda$ is sufficiently large, then

(i) For each $k=0, \ldots, m$, the distance from the boundary of $Q_{k}(h)$ to $Q_{k}(h / 2)$ is larger than $4 h$. In addition, $K\left(p_{k}\right) \cap\left(Q_{k}(7 h / 8) \backslash Q_{k}(3 h / 4)\right)$ contains a square $R_{k}(h)$ of side length larger than $4 h$.

(ii) If two points $p, q$ are on opposite lines of the boundary of $K\left(p_{k}\right)$, and at least one of them is outside $Q_{k}(h)$, then

$$
\operatorname{dist}(p, q) \geq 4 h \text {. }
$$

(iii) Property (ii) also holds for the cone $\widetilde{K}\left(p_{k}\right)$ for $\Omega^{c}$. 
(iv) The square $Q_{k}(h)$ contains the square $Q\left(p_{k}, 2 M h\right)$ centered at $p_{k}$ with sidelength $4 M h$.

(v) If $p$ is a point on $\Gamma_{k}$ and $q$ is a point on $\Gamma_{k+1}$ and at least one of these points is outside of $Q_{k}(h)$, then

$$
\operatorname{dist}(p, q) \geq 4 h \text {. }
$$

Proof. Properties (i)-(iv) clearly hold whenever $\lambda$ is sufficiently large (depending only on the $\alpha_{k}, \beta_{k}, \tilde{\alpha}_{k}$ and $\tilde{\beta}_{k}$ for the cones $\left.K\left(p_{k}\right), \widetilde{K}\left(p_{k}\right)\right)$. We fix a $\lambda$ so that (i)-(iv) hold and show that (v) holds for this $\lambda$. The complement of $K\left(p_{k}\right) \cup \widetilde{K}\left(p_{k}\right)$ has two components $A$ and $B$ each of which is a convex set. The point $p$ is in one of these components and the point $q$ in the other. Suppose, for example, that $p \in A, q \in B$ and $p \notin Q_{k}(h)$ (the other case is the same). The set $A \backslash Q_{k}(h)$ has a boundary which consists of four line segments coming from the sides of the cones $K\left(p_{k}\right)$ and $\widetilde{K}\left(p_{k}\right)$ and the sides of $Q_{k}(h)$. The distance from $A \backslash Q_{k}(h)$ to $B$ is attained at one of the points $a^{*}$ which is at the intersection of one of the sides of the cones with one of the sides of $Q_{k}(h)$. By (ii) and (iii) this distance is larger than $4 h$. Hence, the distance from $p$ to $q$ is also larger than $4 h$.

We now describe a way of covering $\Omega$ near its boundary that we shall use in the sequel. Let $\lambda \geq \max (M, 8)$ be any constant sufficiently large that Proposition 5.1 is valid.

For each $k=1, \ldots, m$, we define $\Gamma_{k}^{\prime}$ as the set of all points on $\Gamma_{k}$ that are not in $Q_{k-1}(h) \cup Q_{k}(h)$. If $k$ is even, we denote by $\Gamma_{k}(h)$ the union of all the open line segments with endpoints $\left(x, \gamma_{k}(x)\right)-h \nu_{k}$ and $\left(x, \gamma_{k}(x)\right)+h \nu_{k}$, with $x \in \Gamma_{k}^{\prime}$. We define $\Gamma_{k}(h)$ in a similar way if $k$ is odd.

Proposition 5.2. Let $\lambda \geq \max (M, 8)$ be any fixed constant so that Proposition 5.1 holds. Then, there is an absolute constant $h_{0}>0$ such that the following holds for all $h \leq h_{0}$.

(i) All the sets $\Gamma_{k}(h), k=1, \ldots, m$, and $Q_{k}(h), k=0, \ldots, m$, are pairwise disjoint. Moreover, the distance between $Q_{k}(h)$ and $Q_{k^{\prime}}(h)$ is larger than $4 h$ whenever $k \neq k^{\prime}$ and the distance between $Q_{k}(h)$ and $\Gamma_{i}(h), i \neq k, k+1$ is larger than $4 h$.

(ii) If $(x, y) \in \Omega$ is not in any of the sets of $(\mathrm{i})$, then the distance of $(x, y)$ to $\Gamma$ is larger than $h / 2 M$ with $M$ the Lipschitz constant for $\Gamma$.

(iii) If $(x, y) \in \Omega$ is in $\Gamma_{k}(h)$, then the distance of $(x, y)$ to $\Gamma_{i}, i \neq k$, is larger than $3 h$.

Proof. We fix $h_{1}$ sufficiently small that $Q\left(p_{k}, h_{1}\right) \cap K\left(p_{k}\right) \subset \Omega, k=0, \ldots, m$. For each $k=1, \ldots, m$, let $\Gamma_{k}^{\prime \prime}$ be the set of all points on $\Gamma_{k}$ that are not in $Q\left(p_{k-1}, h_{1}\right) \cup$ $Q\left(p_{k}, h_{1}\right)$.

We first prove (i). Clearly, if $h$ is small enough, the squares $Q_{k}(h), k=0, \ldots, m$ are pairwise disjoint and the distance between $Q_{k}(h)$ and $Q_{k^{\prime}}(h)$ is larger than $4 h$ whenever $k \neq k^{\prime}$. Moreover, for $h$ sufficiently small, $Q_{k}(h)$ does not intersect any of the sets $\Gamma_{i}(h), i=1, \ldots, m$. By choosing $h$ small enough, we can make the distance between $Q_{k}(h)$ and any of the $\Gamma_{i}(h), i \neq k, k+1$, larger than $4 h$.

It is therefore enough to show that $\Gamma_{k}(h)$ does not intersect any of the sets $\Gamma_{i}(h), i \neq k$. This is clear if $i \neq k-1, k+1$ and $h$ is sufficiently small. For the remaining case, we can assume that $k$ is even (a similar argument applies when $k$ is odd) and check that $\Gamma_{k}(h) \cap \Gamma_{k+1}(h)=\varnothing$ (a similar argument applies for $\left.\Gamma_{k-1}(h)\right)$. If $h$ is sufficiently small (independent of $\left.k\right), Q\left(\Gamma_{k}^{\prime \prime}, h\right)$ is disjoint 
from $\Gamma_{k+1}(h)$. Hence, if $\left(x, \gamma_{k}(x)\right) \in \Gamma_{k}^{\prime \prime}$, the open line segment with endpoints $\left(x, \gamma_{k}(x)\right) \pm h \nu_{k}$ does not intersect $\Gamma_{k+1}(h)$. We are left with considering the case when $\left(x, \gamma_{k}(x)\right) \in \Gamma_{k}^{\prime} \backslash \Gamma_{k}^{\prime \prime}$ and $\left(\gamma_{k+1}(y), y\right) \in \Gamma_{k+1}^{\prime}$ and showing that the line segment with endpoints $\left(x, \gamma_{k}(x)\right) \pm h \nu_{k}$ does not intersect the line segment with endpoints $\left(\gamma_{k+1}(y), y\right) \pm h \nu_{k+1}$. Since $\left(x, \gamma_{k}(x)\right)$ is not in $Q_{k}(h)$, we find from Proposition [5.1 that the distance from $\left(x, \gamma_{k}(x)\right)$ to $\left(\gamma_{k}(y), y\right)$ is larger than $4 h$. It follows that the line segment with endpoints $\left(x, \gamma_{k}(x)\right) \pm h \nu_{k}$ does not intersect the line segment with endpoints $\left(\gamma_{k+1}(y), y\right) \pm h \nu_{k+1}$. Therefore we have verified (i).

We next prove (ii). Let $(x, y) \in \Omega$ be a point that is not in any of the sets of (i). We shall estimate the distance from $(x, y)$ to the curve $\Gamma_{k}$ for each $k$. We can again without loss of generality assume that $k$ is even and that $x_{k}>x_{k-1}$. Let $\left(z, \gamma_{k}(z)\right)$ be the point on $\Gamma_{k}$ which is closest to $(x, y)$. If $z \in\left[x_{k-1}, x_{k-1}+h / 2\right] \cup$ $\left[x_{k}-h / 2, x_{k}\right]$, then from the Lipschitz condition for $\gamma_{k}$, it follows that $\left(x, \gamma_{k}(z)\right) \in$ $Q\left(p_{k-1}, M h / 2\right) \cup Q\left(p_{k}, M h / 2\right)$. Since $(x, y)$ is not in $Q_{k-1}(h) \cup Q_{k}(h)$ and (iv) of Proposition 5.1 gives that this latter set contains $Q\left(p_{k-1}, M h\right) \cup Q\left(p_{k}, M h\right)$. Using the fact that $\lambda>M$, we have that the distance between $\left(z, \gamma_{k}(z)\right)$ and $(x, y)$ is larger than $M h / 2$ as desired. We can therefore assume for the remainder of the proof of (ii) that $z \in\left[x_{k-1}+h / 2, x_{k}-h / 2\right]$. If $|x-z| \geq h / 2 M$, there is nothing to prove. On the other hand if $|x-z| \leq h / 2 M$, then $\left(x, \gamma_{k}(x)\right)$ is a point on $\Gamma_{k}$ and $\left|\gamma_{k}(x)-\gamma_{k}(z)\right| \leq h / 2$. Hence,

$$
\left|y-\gamma_{k}(z)\right| \geq\left|y-\gamma_{k}(x)\right|-h / 2 .
$$

We claim that $\left|y-\gamma_{k}(x)\right| \geq h$ which will complete the proof. Since $\left(x, \gamma_{k}(x)\right)$ is not in $Q_{k-1}(h) \cup Q_{k}(h)$, we have that $\left(x, \gamma_{k}(x)\right) \in \Gamma_{k}^{\prime}$. But then our claim follows because $(x, y)$ is not in $\Gamma_{k}(h)$.

Finally, we prove (iii). If $h$ is sufficiently small this property will hold for $i \neq$ $k-1, k+1$ because the curves $\Gamma_{i}$ are disjoint from $\Gamma_{k}$ for $i \neq k-1, k, k+1$. We shall prove that the distance from $(x, y)$ to $\Gamma_{k+1}$ is larger than $3 h$. A similar proof applies for $k-1$. We shall also assume that $k$ is even; a similar proof applies when $k$ is odd. Since $\left(x, \gamma_{k}(x)\right)$ is not in $Q_{k}(h)$, we have from Proposition 5.1(v) that the distance of $\left(x, \gamma_{k}(x)\right)$ to $\Gamma_{k+1}$ is larger than $4 h$. It follows that the distance from $(x, y)$ to $\Gamma_{k+1}$ is larger than $3 h$.

We shall next define for each dyadic level $j$, a collection of cells $\mathcal{C}_{j}$ which will satisfy the properties (C1)-(C5). Let $h_{j}:=\lambda 2^{-j}$ where $\lambda$ is large enough to satisfy our previous conditions and in addition $\lambda \geq 64 L M N(L+M+N)^{2}$ where $L$ is the integer of the previous sections corresponding to the support of $\varphi, M$ is the Lipschitz constant, and $N$ denotes the degree of polynomials contained in the span of the shifts of $\varphi$. We let $j_{0} \geq 0$ be the smallest integer such that $h_{j} \leq h_{0}$ with $h_{0}$ the number given in Proposition 5.2 We shall construct a partition of $\Omega_{j}$ into a collection of cells $\mathcal{C}_{j}$ for all $j \geq j_{0}$.

There are three types of cells in $\mathcal{C}_{j}$. Let $\mathcal{L}_{j}:=2^{-j} \mathbb{Z}^{2}$ and let $\Omega_{j}$ be defined as earlier.

Point cells: If $\gamma$ is a lattice point in $\Omega \cap \mathcal{L}_{j}$ which is not in any of the sets $\Gamma_{k}\left(h_{j}\right), k=1, \ldots, m$, or $Q_{k}\left(h_{j}\right), k=0, \ldots, m$, then we put the 0-dimensional cells $C=\{\gamma\}$ into $\mathcal{C}_{j}$. Each such cell corresponds to the direction set $I=\varnothing$ and has representer $\kappa=\gamma$. The set $G(C)=C$ in this case.

Towers: Let $k=1, \ldots, m$, and suppose that $\gamma \in \Omega_{j}$ is in the set $\Gamma_{k}\left(h_{j}\right)$ with direction vector $\nu_{k}$. Let $l$ be the largest nonnegative integer such that $\gamma-$ 
$2^{-j} l \nu_{k}$ is in $\Gamma_{k}\left(h_{j}\right)$. We define $\kappa:=\gamma-2^{-j}(l-N-L) \nu_{k}$ and define the onedimensional cell $C$ with representer $\kappa$ as the set of all lattice points $\kappa+i 2^{-j} \nu_{k}, i=$ $-(N+L),-(N+L)+1, \ldots$, that are in $\Omega_{j}$. This cell $C$ has associated to it the set of direction indices $I$ which is $I=\{1\}$ in the case $k$ is odd and $I=\{2\}$ in the case that $k$ is even. In this case, $\sigma$ is defined to be the nonzero component in $\nu_{k}$. The set $G(C)$ is the set of all points $\kappa+i 2^{-j} \nu_{k}, i=0, \ldots, N$. Because $h_{j}=\lambda 2^{-j}$ and $\lambda \geq 4(N+L)$, it follows that $G(C) \subset \Omega$. Also $\operatorname{dist}(\kappa, \Gamma) \leq c 2^{-j}$.

Corners: For each $k=0, \ldots, m$, we let $C$ be the cell consisting of all lattice points in $Q_{k}\left(h_{j}\right) \cap \Omega_{j}$. For each such cell $I=\{1,2\}$ and $\sigma=\nu_{k-1}+\nu_{k}$. By Proposition 5.1 (i), there is a square $R_{k}\left(h_{j}\right)$ of sidelength $4 h_{j}$ with

$$
R_{k}\left(h_{j}\right) \subset K\left(p_{k}\right) \cap\left(Q_{k}\left(7 h_{j} / 8\right) \backslash Q_{k}\left(3 h_{j} / 4\right)\right) .
$$

We take a lattice point $\kappa \in R_{k}$ which is most central to $R_{k}\left(h_{j}\right)$ and define $\kappa$ as the representer of $C$ and $G(C):=\left\{\kappa+2^{-j}\left(i \nu_{k}+i^{\prime} \nu_{k+1}\right): 0 \leq i, i^{\prime} \leq N\right\}$. Then, clearly $G(C) \subset \Omega$ and even $[G(C)]_{j} \subset R_{k}\left(h_{j}\right)$. Moreover, we shall often make use of the fact that the distance of $G(C)$ to $\Gamma$ is $\geq 3 h_{j}$ which follows easily from the facts that $R_{k}\left(h_{j}\right) \subset \Omega, \kappa$ is most central to $R_{k}\left(h_{j}\right)$ and $L+N+1 \leq \lambda / 16$. It follows also that $\operatorname{dist}(\kappa, \Gamma) \leq c 2^{-j}$.

We now proceed to show that the sets of cells $\mathcal{C}_{j}, j \geq j_{0}$, satisfy the conditions $(\mathrm{C} 1-5)$.

(C1) Let $C \in \mathcal{C}_{j}$. We have already shown that $G(C) \subset \Omega$ when we defined $G(C)$. To verify $(\mathrm{C} 1)$, we need to show that $[G(C)] \subset \Omega$ with $[\cdot]:=[\cdot]_{j}$.

If $C=\{\kappa\}$ is a point cell, then $[G(C)]=[\{\kappa\}]$ and $\operatorname{dist}(\kappa, \Gamma) \geq h_{j} / 2 M$ because of (ii) of Proposition 5.2. Since $h_{j}=\lambda 2^{-j}$ and $\lambda \geq 4 L M$, $[G(C)] \subset \Omega$.

Next, assume that $C \in \mathcal{C}_{j}$ is a tower associated to $\Gamma_{k}(h)$ which has the representer $\kappa$ and the direction set $I$. Then, $G(C)=\left\{\kappa-i 2^{-j} \nu_{k}, i=0, \ldots, N\right\}$ and we thus have $[G(C)] \subset \kappa+2^{-j}[-L-N, L+N]^{2}$. Because of (iii) in Proposition $5.2[G(C)]$ does not intersect any set $\Gamma_{i}, i \neq k$. We now check that $[G(C)]$ does not intersect $\Gamma_{k}$. Suppose that $\kappa=(x, y)$ and $\left(z, \gamma_{k}(z)\right)$ is any point on $\Gamma_{k}$. We want to show that $\left(z, \gamma_{k}(z)\right)$ is not in $[G(C)]$. If $|x-z|>(N+L) 2^{-j}$, then this is clear. If $|x-z| \leq(N+L) 2^{-j}$, then $\left|\gamma_{k}(x)-\gamma_{k}(z)\right| \leq M(N+L) 2^{-j}$ because $\gamma_{k}$ satisfies a Lipschitz condition with constant $M$. It follows that

$$
\begin{aligned}
\left|y-\gamma_{k}(z)\right| & \geq\left|y-\gamma_{k}(x)\right|-\left|\gamma_{k}(x)-\gamma_{k}(z)\right| \\
& \geq h_{j}-(N+L+1) 2^{-j}-M(N+L) 2^{-j} \geq h_{j} / 2
\end{aligned}
$$

because $h_{j}=\lambda 2^{-j}$ and $\lambda \geq 4 M N L\left(M+N_{L}\right)^{2}$. Since $h_{j} \geq 2(N+L) 2^{-j}$, we see that $\left(z, \gamma_{k}(z)\right)$ is not in $[G(C)]$ and therefore condition $(\mathrm{C} 1)$ holds for towers.

For corners, we have already noted that $[G(C)]_{j} \subset \Omega$.

(C2) Suppose that $\mu \in \Omega_{j+1}$ satisfies $[\{\mu\}]_{j+1} \subset\left[G_{j}\right]_{j}$. We need to show that $\mu \in G_{j+1}$. We shall show that $\{\mu\}$ is a point cell in $\mathcal{C}_{j+1}$ and therefore $\mu \in G_{j+1}$. By assumption, there is a cell $C \in \mathcal{C}_{j}$ and a point $\zeta \in G(C)$ such that $\mu \in[\{\zeta\}]_{j}$. There is also a cell $C^{\prime} \in \mathcal{C}_{j+1}$ which contains $\mu$. We consider the various possibilities for $C$ and $C^{\prime}$ with $c^{\prime}$ not a point cell and derive a contradiction in each case.

$C$ is a corner and $C^{\prime}$ is a corner: Let $C$ correspond to the point $p_{k}$ and $C^{\prime}$ correspond to the point $p_{k^{\prime}}$. If $k \neq k^{\prime}$, then $C^{\prime} \subset Q_{k^{\prime}}\left(h_{j}\right)$ and by Proposition 5.2 (i), the distance between $Q_{k}\left(h_{j}\right)$ and $Q_{k^{\prime}}\left(h_{j}\right)$ is larger than $4 h_{j}$. Hence, we arrive at the contradiction $\mu \notin[\zeta]_{j}$. On the other hand, if $k=k^{\prime}$, then we have already noted that $[G(C)]_{j} \subset R_{k}\left(h_{j}\right) \subset Q_{k}\left(7 h_{j} / 8\right) \backslash Q_{k}\left(3 h_{j} / 4\right)$. Since $C^{\prime} \subset Q_{k}\left(h_{j} / 2\right)$, we again arrive at the contradiction $\mu \notin[\zeta]_{j}$. 
$C$ is a corner and $C^{\prime}$ is a tower: Let $C$ be a corner corresponding to the point $p_{k}$. We have observed at the definition of $G(C)$ that $\operatorname{dist}(G(C), \Gamma) \geq 3 h_{j}$. Since $\operatorname{dist}(\mu, \Gamma) \leq h_{j} / 2$, it follows that $\mu$ and $\zeta$ are at least a distance $h_{j}$ apart. Since $h_{j} \geq 2 L 2^{-j}$ this contradicts the assumption that $\mu \in[\{\zeta\}]_{j}$.

$C$ is a tower and $C^{\prime}$ is a corner: Let $C^{\prime}$ correspond to the point $p_{k^{\prime}}$. We have $\mu \in C^{\prime} \subset Q_{k^{\prime}}\left(h_{j} / 2\right)$ and $\zeta \notin Q_{k^{\prime}}\left(h_{j}\right)$. Hence, by Proposition [5.1] (i), $\operatorname{dist}(\mu, \zeta) \geq$ $4 h_{j} \geq 2 L 2^{-j}$. Thus, we have the contradiction that $\mu \notin[\{\zeta\}]_{j}$.

$C$ is a tower and $C^{\prime}$ is a tower: We can assume that $C^{\prime}$ is a tower associated to $\Gamma_{k^{\prime}}\left(h_{j+1}\right)$ with $k^{\prime}$ even; the proof in the case that $k$ is odd is the same. Let $C$ be a tower associated with $\Gamma_{k}\left(h_{j}\right)$. We consider first the possibility that $k \neq k^{\prime}$. Since $\mu \in \Gamma_{k^{\prime}}\left(h_{j+1}\right)$, the distance from $\mu$ to $\Gamma_{k^{\prime}}$ is at most $h_{j+1}=h_{j} / 2$. From Proposition 5.2 part (iii), $\operatorname{dist}\left(\zeta, \Gamma_{k^{\prime}}\right) \geq 3 h_{j}$. Hence, the distance between $\mu$ and $\zeta$ is at least $2 h_{j}$. Since $h_{j}=\lambda 2^{-j}$ and $\lambda \geq 2 L$, we see that it is not possible for $\mu$ to be in $[\zeta]_{j}$ and we arrive at our contradiction.

The remaining possibility is that $k=k^{\prime}$. We can write $\mu=\left(z, \gamma_{k}(z)\right)-l^{\prime} 2^{-j} \nu_{k}$ with $\nu_{k}$ the (exterior) direction vector for $\Gamma_{k}$ and $l^{\prime} \leq \lambda / 2$ and similarly, we can write $\zeta=\left(x, \gamma_{k}(x)\right)-l 2^{-j} \nu_{k}$. Since $\zeta \in G(C)$, we have

$$
l \geq \lambda-2(N+l+1)
$$

(see the definition of $G(C)$ for towers). In order for $\mu$ to be in $[\{\zeta\}]_{j}$, we must have $|x-z| \leq L 2^{-j}$ and $\left|\gamma_{k}(x)-l 2^{-j}-\left(\gamma_{k}(z)\right)-l^{\prime} 2^{-j}\right| \leq L 2^{-j}$. Also from the Lipschitz condition for $\gamma_{k}$, it follows that $\left|\gamma_{k}(x)-\gamma_{k}(z)\right| \leq M|x-z| \leq M L 2^{-j}$. With this, we find that

$$
\left|l^{\prime} 2^{-j}-l 2^{-j}\right| \leq(M L+L) 2^{-j}
$$

Therefore,

$$
l \leq l^{\prime}+M L+L \leq \lambda / 2+M L+L .
$$

But since $\lambda>16 M L N(M+L+N)$, this contradicts (5.3).

$C$ is a point cell and $C$; is a corner: Let $C^{\prime}$ correspond to $p_{k^{\prime}}$. Then, $\mu \in C^{\prime} \subset Q_{k^{\prime}}\left(h_{j} / 2\right)$. On the other hand, $\zeta$ is not in $Q_{k^{\prime}}\left(h_{j}\right)$. From Proposition 5.1 (i), $\operatorname{dist}(\zeta, \mu) \geq 4 h_{j}$. Since $h_{j}=\lambda 2^{-j}$ and $\lambda \geq L$, this contradicts that $\mu \in[\{\zeta\}]_{j}$.

$C$ is a point cell and $C^{\prime}$ is a tower: Let $C^{\prime}$ correspond to $\Gamma_{k^{\prime}}\left(h_{j+1} / 2\right)$, with $k^{\prime}$ even and $x_{k^{\prime}-x}<x_{k^{\prime}}$; the other cases are handled in the same way. Then, there is a point $\left(z, \gamma_{k^{\prime}}(z)\right)$ with $x_{k^{\prime}-1}+h_{j} / 2 \leq z \leq x_{k^{\prime}}-h_{j} / 2$ such that $\mu$ is on the line segment with end points $\left(z, \gamma_{k^{\prime}}(z)\right) \pm h_{j} / 2 \nu_{k^{\prime}}$. Let $C=\{\zeta\}$ with $\zeta=(x, y)$. If $|x-z|>l 2^{-j}$, then $\mu \notin[\{\zeta\}]_{j}$. On the other hand, if $|x-z| \leq L 2^{-j}$, then $\left|\gamma_{k^{\prime}}(x)-\gamma_{k^{\prime}}(z)\right| \leq M L 2^{-j}$. Therefore, if $\mu$ is to be in $[\{\zeta\}]_{j}$, then

$$
\left|y-\gamma_{k^{\prime}}(x)\right| \leq\left|y-\gamma_{k^{\prime}}(z)\right|+M L 2^{-j} \leq h_{j} / 2+L 2^{-j}+M L 2^{-j}<h_{j} .
$$

But this contradicts the fact that $(x, y)$ is not in any of the sets $\Gamma_{k^{\prime}}\left(h_{j}\right), Q_{k^{\prime}-1}\left(h_{j}\right)$ or $Q_{k^{\prime}}\left(h_{j}\right)$.

This completes the verification of Property (C2).

(C3) Let $C \in \mathcal{C}_{j}(I, \sigma)$ and $C^{\prime} \in \mathcal{C}_{j+1}\left(I^{\prime}, \sigma^{\prime}\right)$, we need to show that if $[C]_{j} \cap C^{\prime} \neq \varnothing$, then $I^{\prime} \subseteq I$. If $C$ is a corner or if $C^{\prime}$ is a point cell, then this is obvious. We consider the remaining possibilities.

$C$ is a point cell or a tower and $C^{\prime}$ is a corner: Let $C^{\prime}$ be a corner for $p_{k^{\prime}}$. Then $C^{\prime} \subset Q_{k^{\prime}}\left(h_{j} / 2\right)$ while $C$ is in the complement of $Q_{k^{\prime}}\left(h_{j}\right)$. Hence, from Proposition [5.1 (i), the distance between any point in $C$ and any point in $C^{\prime}$ is larger than $4 h_{j}$. Since $h_{j}=\lambda 2^{-j}$ and $\lambda h_{j} \geq L 2^{-j},[C]_{j} \cap C^{\prime}=\varnothing$. 
$C$ is a tower and $C^{\prime}$ is a tower: Let $C$ be associated to $\Gamma_{k}\left(h_{j}\right)$ and $C^{\prime}$ be associated to $\Gamma_{k^{\prime}}\left(h_{j+1}\right)$. If $k=k^{\prime}$, then $I=I^{\prime}$. If $k=k^{\prime}$, then according to Proposition 5.2 (iii), any point in $C$ has distance at least $3 h_{j}$ from $\Gamma_{k^{\prime}}$. Since $h_{j}=\lambda 2^{-j}$ and $\lambda \geq L$, it follows that any point in $[C]_{j}$ has distance at least $2 h_{j}$ from $\Gamma_{k^{\prime}}$. Since any point in $C^{\prime}$ has distance at most $h_{j+1} \leq h_{j}$ from $\Gamma_{k^{\prime}}$, we have that $[C]_{j} \cap C^{\prime}=\varnothing$.

$C$ is a point cell and $C^{\prime}$ is a tower: This proof is similar to the corresponding case in the proof of $(\mathrm{C} 2)$. Let $C^{\prime}$ be associated to $\Gamma_{k^{\prime}}\left(h_{j+1}\right)$. We can assume that $k^{\prime}$ is even; the case $k^{\prime}$ is odd is proved similarly. Let $C^{\prime}$ be associated to the point $\left(z, \gamma_{k^{\prime}}(z)\right)$ on $\Gamma_{k^{\prime}}$. We also assume that $x_{k^{\prime}-1}<x_{k^{\prime}}$; the other case is handled similarly. Using (iv) of Proposition 5.1 (as in previous arguments in this section), it follows that $x_{k^{\prime}-1}+h_{j+1} \leq z \leq x_{k^{\prime}}+h_{j+1}$. Let $C=\{\zeta\}$ with $\zeta=(x, y)$. If $|x-z|>L 2^{-j},[C]_{j} \cap C^{\prime}=\varnothing$. If $|x-z| \leq L 2^{-j}$, then $\left(x, \gamma_{k}(x)\right)$ is a point on $\Gamma_{k}$. By the Lipschitz condition for $\gamma_{k^{\prime}}$, we have $\left|\gamma_{k^{\prime}}(x)-\gamma_{k^{\prime}}(z)\right| \leq M L 2^{-j}$. Now the point $\left(x, \gamma_{k^{\prime}}(x)\right)$ is either in $\Gamma_{k^{\prime}}\left(h_{j}\right)$ or in $Q_{k^{\prime}-1}\left(h_{j}\right) \cup Q_{k^{\prime}}\left(h_{j}\right)$. By the definition of these sets $\left|y-\gamma_{k^{\prime}}(x)\right| \geq h_{j}$. Hence

$$
\left|y-\gamma_{k^{\prime}}(z)\right| \geq h_{j}-M l 2^{-j}
$$

On the other hand, since $[\{\zeta\}]_{j}$ contains points of $C^{\prime}$, we must have

$$
\left|y-\gamma_{k^{\prime}}(z)\right| \leq h_{j} / 2+L 2^{-j}
$$

This last inequality contradicts (5.4) because $h_{j}=\lambda 2^{-j}$ and $\lambda>2(M L+L)$.

We have verified (C3).

(C4) Let $C \in \mathcal{C}_{j}(I, \sigma)$ and $C^{\prime} \in \mathcal{C}_{j}\left(I^{\prime}, \sigma^{\prime}\right)$ be two cells from $\mathcal{C}_{j}$ with $C \neq C^{\prime}$ and

$$
[C, I] \cap\left[C^{\prime}, I\right]=\varnothing .
$$

We need to show that $I^{\prime} \subset I, I^{\prime} \neq I$. We consider the following cases.

$C$ is a corner and $C^{\prime}$ is a corner: If $C$ and $C^{\prime}$ correspond to different points $p_{k}$, then (5.5) is not satisfied because of Proposition 5.2 (i).

$C$ is a corner and $C^{\prime}$ is a tower or a point cell: In this case, it is obvious that $I^{\prime} \subset I$ and $I^{\prime} \neq I$.

$C$ is a tower and $C^{\prime}$ is a corner: Let $C$ correspond to $\Gamma_{k}\left(h_{j}\right)$ and let $C^{\prime}$ correspond to the point $p_{k^{\prime}}$ so that $C^{\prime} \subset Q_{k^{\prime}}\left(h_{j}\right)$. If $k \neq k^{\prime}, k^{\prime}+1$, then (5.5) is not satisfied because of Proposition $5.2(\mathrm{i})$. We consider the case $k=k^{\prime}$ and $k$ even; the other remaining cases are similar. Let $C$ correspond to the point $\left(x, \gamma_{k}(x)\right)$ from $\Gamma_{k}\left(h_{j}\right)$. Then $[C, I]$ is contained in the line segment with endpoints $\left(x, \gamma_{k}(x)\right) \pm\left(h_{j}+L 2^{-j}\right) \nu_{k}$. This line segment is disjoint from $Q_{k}\left(h_{j}\right)$ by the definition of $Q_{k}\left(h_{j}\right)$.

$C$ is a tower and $C^{\prime}$ is a tower: Let $C$ correspond to $\Gamma_{k}\left(h_{j}\right)$ and $C^{\prime}$ correspond to $\Gamma_{k^{\prime}}\left(h_{j}\right)$. If $k=k^{\prime}$, then (5.5) is not satisfied by the definition of towers. If $k \neq k^{\prime}$, then (5.5) is again not satisfied because of Proposition 5.2 (iii).

$C$ is a tower and $C^{\prime}$ is a point cell: This is obvious since $I^{\prime}=\varnothing$ in this case.

$C$ is a point cell and $C^{\prime}$ is a corner, tower, or point cell: In this case $I=\varnothing$ and therefore $[C, I]=C$ and $\left[C^{\prime}, I^{\prime}\right]=C^{\prime}$ and hence (5.5) is not satisfied because of the disjointness of the cells in $\mathcal{C}_{j}$.

We have verified property (C4).

(C5) This property is obvious from the definition of the cells. 


\section{REFERENCES}

[A] R. Adams, Sobolev Spaces, Academic Press, New York, 1975. MR 56:9247

[BL] J. Bergh and J. Löfstrom, Interpolation Spaces, an Introduction, Grundlehren der Mathematischen Wissenschaften 223, Springer, 1986. MR 58:2349]

[BDR] C. de Boor, R. A. de Vore and A. Ron, On the construction of multivariate (pre) wavelets, Constructive Approximation 9 (1993), 123-166. MR 94k:41048

[CDP] J. M. Carnicer, W. Dahmen and J. M. Peña, Local decomposition of refinable spaces, in preparation.

[CDM] A. S. Cavaretta, W. Dahmen and C. A. Micchelli, Stationary Subdivision, Mem. Amer. Math. Soc., Vol. 93, No. 453 (1991). MR 92h:65017

[CQ] C. K. Chui and E. Quak, Wavelets on a bounded interval, in Numerical Methods of Approximation Theory, Vol. 9, D. Braess and L. L. Schumaker, eds., International Series of Numerical Mathematics, Vol. 105, Birkhäuser, Basel, 1992. MR 95b:42027

[CD] A. Cohen and I. Daubechies, Nonseparable bidimensional wavelet bases, Rev. Mat. Iberoamericana 9 (1993), 51-137. MR 94k:42047

[CDF] A. Cohen, I. Daubechies and J.-C. Feauveau, Biorthogonal bases of compactly supported wavelets, Comm. Pure Appl. Math. 45 (1992), 485-560. MR 93e:42044

[CDV] A. Cohen, I. Daubechies and P. Vial, Wavelets and fast wavelet transforms on the interval, ACHA 1 (1994), 54-81. MR 94m:42074

[D] W. Dahmen, Some remarks on multiscale transformations, stability and biorthogonality, in Curves and surfaces, P. J. Laurent, A. Le Méhauté, L. L. Schumaker, eds., Academic Press, 1994. MR 92c:65006

[D2] W. Dahmen, Stability of multiscale transformations, preprint, Fourier Anal. Appl. 2 (1996), 341-361. MR 97ki:46133

[DDS] W. Dahmen, R. A. DeVore and K. Scherer, Multidimensional spline approximation, SIAM J. Numer. Anal. 17 (1980), 380-402. MR 81j:41015

[DK] W. Dahmen and A. Kunoth, Multilevel preconditioning, Numer. Math. 63 (1992), 315344. MR 93j:65065

[DM93] W. Dahmen, C. A. Micchelli, Biorthogonal wavelet expansions, Constructive Approximation 13 (1997), 293-328. MR 99c:39039

[Dau] I. Daubechies, Orthonormal bases of compactly supported wavelets, Comm. Pure Appl. Math. 41 (1988), 909-996. MR 90m:42039

[Dau2] I. Daubechies, Ten lectures on wavelets, CBMS-NSF Regional Conference Series in Applied Math., 61, SIAM, 1992. MR 93e:42045

[DJP] R. DeVore, B. Jawerth and V. Popov, Compression of wavelet decompositions, Amer. J. Math. 114 (1992), 737-785. MR 94a:42025

[DL] R. DeVore and G. Lorentz, Constructive Approximation, Grundlehren der Mathematischen Wissenschaften, Springer, 1993. MR 95f:41001

[DP] R. DeVore and V. Popov, Interpolation of Besov spaces, Trans. Amer. Math. Soc. 305 (1988), 397-414. MR 89h:46044

[DS] R. DeVore and R. Sharpley, Maximal functions measuring smoothness, Mem. Amer. Math. Soc., No. 293, 1983. MR 85g:46039

[JM] R. Q. Jia and C. A. Micchelli, Using the refinement equation for the construction of pre-wavelets II: Powers of two, in Curves and Surfaces, P. J. Laurent, A. LeMéhauté, L. L. Schumaker, eds., Academic Press, New York, 1991, pp. 209-246. MR 93e:65024

[JS] H. Johnen and K. Scherer, On the equivalence of the $K$-functional and moduli of continuity and some applications, in Constructive Theory of Functions of Several Variables, Lecture Notes in Math., Vol. 571, Springer, 1977, pp. 119-140. MR 58:7060

[LM] P. G. Lemarié and G. Malgouyres, Support des fonctions de base dans une analyse multiresolution, C. R. Acad. Sci. Paris 213 (1991), 377-380. MR 92k:42044

[M] V. G. Maz'ja, Sobolev Spaces, Springer-Verlag, Berlin, 1985. MR 87g:46056

[Me] Y. Meyer, Ondelettes et Opérateurs, Vols. 1 and 2, Hermann, Paris, 1990. MR 93i:42002, MR 93i:42003

[OS] P. Oswald and E. A. Storozhenko, Jackson's theorem in the spaces $L_{p}\left(\mathbb{R}^{k}\right), 0<p<1$, Siberian. Math. 19 (1978), 630-639. MR 58:12154

[Sch] I. J. Schoenberg, Contribution to the problem of approximation of equidistant data by analytic functions, Quart. Appl. Math. 4 (1946), 45-99, 112-141. MR 7:487b MR 8:55d 
[S] E. Stein, Singular Integrals and Differentiability Properties of Functions, Princeton University Press, Princeton, N.J., 1970.

[T] H. Triebel, Interpolation Theory Function Spaces and Differential Operators, NorthHolland, Amsterdam, 1978. MR 80i:46032b

Laboratoire d'Analyse Numerique, Université de Paris Vi, 4 Place Jussieu, 75005 PARIS, France

E-mail address: cohen@ann.jussieu.fr

Rheinisch-Westf Technische Hochscule, Templergraben 55, D-52052 Aachen, GerMANY

Industrial Mathematics Institute, University of South Carolina, Columbia, South Carolina 29208-0001

E-mail address: devore@math.sc.edu 